\title{
Sufficient and necessary conditions for $C P$ conservation in the case of degenerate Majorana neutrino masses
}

\author{
Bingrong Yu $\odot^{\dagger}$ and Shun Zhou $\odot^{*}$ \\ Institute of High Energy Physics, Chinese Academy of Sciences, Beijing 100049, China \\ and School of Physical Sciences, University of Chinese Academy of Sciences, Beijing 100049, China
}

(Received 9 December 2020; accepted 12 January 2021; published 19 February 2021)

\begin{abstract}
We carry out a systematic study of the sufficient and necessary conditions for $C P$ conservation in the leptonic sector with massive Majorana neutrinos. In particular, the emphasis is placed on the number of $C P$-violating phases in the presence of a partial mass degeneracy (e.g., $m_{1}=m_{2} \neq m_{3}$ ) or a complete mass degeneracy $m_{1}=m_{2}=m_{3}$, where $m_{i}$ (for $i=1,2,3$ ) stand for the masses of three ordinary neutrinos. In the canonical seesaw model with three right-handed neutrino singlets, $C P$-violating phases in the special case of a partial (e.g., $M_{1}=M_{2} \neq M_{3}$ ) or complete (i.e., $M_{1}=M_{2}=M_{3}$ ) mass degeneracy of three heavy Majorana neutrinos are also examined. In addition, we derive the renormalization-group equations of the weak-basis invariants in the effective theory with a general mass spectrum of Majorana neutrinos, to which the solutions establish the direct connection between $C P$ violation at low- and high-energy scales.
\end{abstract}

DOI: 10.1103/PhysRevD.103.035017

\section{INTRODUCTION}

Neutrino oscillation experiments have firmly established that neutrinos are indeed massive and lepton flavors are significantly mixed $[1,2]$. One main goal of future longbaseline accelerator neutrino oscillation experiments is to discover $C P$ violation in the leptonic sector and precisely measure the relevant $C P$-violating phase [3]. To account for tiny neutrino masses, one can go beyond the standard model (SM) by introducing three right-handed neutrino singlets $N_{i \mathrm{R}}$ (for $i=1,2,3$ ). Then the $\mathrm{SU}(2)_{\mathrm{L}} \times \mathrm{U}(1)_{\mathrm{Y}}$ gauge-invariant Lagrangian for lepton masses, flavor mixing, and $C P$ violation can be written as

$\mathcal{L}_{\text {lepton }}=-\bar{\ell}_{\mathrm{L}} Y_{l} l_{\mathrm{R}} H-\bar{\ell}_{\mathrm{L}} Y_{\nu} \tilde{H} N_{\mathrm{R}}-\frac{1}{2} \overline{N_{\mathrm{R}}^{\mathrm{C}}} M_{\mathrm{R}} N_{\mathrm{R}}+$ H.c.,

where $\ell_{\mathrm{L}} \equiv\left(\nu_{\mathrm{L}}, l_{\mathrm{L}}\right)^{\mathrm{T}}$ and $\tilde{H} \equiv \mathrm{i} \sigma_{2} H^{*}$, with $H \equiv\left(\varphi^{+}, \varphi^{0}\right)^{\mathrm{T}}$, are the left-handed lepton doublet and the Higgs doublet, $Y_{l}$ and $Y_{\nu}$ are the charged-lepton and Dirac neutrino Yukawa coupling matrices, and $M_{\mathrm{R}}$ is the Majorana mass matrix for right-handed neutrino singlets. Note that $N_{\mathrm{R}}^{\mathrm{C}} \equiv \mathcal{C}{\overline{N_{\mathrm{R}}}}^{\mathrm{T}}$ has

*Corresponding author.

zhoush@ihep.ac.cn

yubr@ihep.ac.cn

Published by the American Physical Society under the terms of the Creative Commons Attribution 4.0 International license. Further distribution of this work must maintain attribution to the author(s) and the published article's title, journal citation, and DOI. Funded by SCOAP ${ }^{3}$. been defined with $\mathcal{C} \equiv \mathrm{i} \gamma^{2} \gamma^{0}$ being the charge-conjugation matrix. As the Higgs field acquires its vacuum expectation value $\left\langle\varphi^{0}\right\rangle=v / \sqrt{2}$ with $v \approx 246 \mathrm{GeV}$ and the gauge symmetry is spontaneously broken down, the charged-lepton mass matrix and the Dirac neutrino mass matrix are then given by $M_{l} \equiv Y_{l} v / \sqrt{2}$ and $M_{\mathrm{D}} \equiv Y_{\nu} v / \sqrt{2}$, respectively.

In such a minimal extension of the SM, three ordinary neutrinos are massive Majorana particles, namely, they are their own antiparticles $[4,5]$. The lepton mass spectrum, flavor mixing, and $C P$ violation at the low-energy scale are then governed by the following effective Lagrangian:

$$
\mathcal{L}_{\text {lepton }}^{\prime}=-\overline{l_{\mathrm{L}}} M_{l} l_{\mathrm{R}}-\frac{1}{2} \overline{\nu_{\mathrm{L}}} M_{\nu} \nu_{\mathrm{L}}^{\mathrm{C}}+\frac{g}{\sqrt{2}} \overline{l_{\mathrm{L}}} \gamma^{\mu} \nu_{\mathrm{L}} W_{\mu}^{-}+\text {H.c. }
$$

where the effective mass matrix for three light Majorana neutrinos is given by the famous seesaw formula $M_{\nu}=$ $-M_{\mathrm{D}} M_{\mathrm{R}}^{-1} M_{\mathrm{D}}^{\mathrm{T}}$ [6-10], which is in general complex and symmetric, and the last term stands for the charged-current weak interaction, with $g$ being the gauge coupling constant of the $\mathrm{SU}(2)_{\mathrm{L}}$ gauge group. As the Majorana mass term of right-handed neutrinos is not subject to the spontaneous gauge symmetry breaking, the smallness of light Majorana neutrino masses $\mathcal{O}\left(M_{\nu}\right) \lesssim 0.1 \mathrm{eV}$ can be ascribed to the largeness of heavy Majorana neutrino masses $\mathcal{O}\left(M_{\mathrm{R}}\right) \gtrsim 10^{14} \mathrm{GeV}$, with $\mathcal{O}\left(M_{\mathrm{D}}\right) \sim 10^{2} \mathrm{GeV}$. After diagonalizing the lepton mass matrices via $V_{l}^{\dagger} M_{l} V_{l}^{\prime}=$ $\hat{M}_{l} \equiv \operatorname{diag}\left\{m_{e}, m_{\mu}, m_{\tau}\right\} \quad$ and $V_{\nu}^{\dagger} M_{\nu} V_{\nu}^{*}=\hat{M}_{\nu} \equiv \operatorname{diag}\left\{m_{1}\right.$, $\left.m_{2}, m_{3}\right\}$, where $V_{l}, V_{l}^{\prime}$, and $V_{\nu}$ are $3 \times 3$ unitary matrices, 
and converting into the mass basis, we can obtain the leptonic flavor mixing matrix or the Pontecorvo-MakiNakagawa-Sakata (PMNS) matrix $U=V_{l}^{\dagger} V_{\nu} \quad[11,12]$, which then appears in the charged-current interaction as the origin of lepton flavor mixing and $C P$ violation.

Since the discovery of leptonic $C P$ violation is the primary goal of future neutrino oscillation experiments and it may also be connected to cosmological matter-antimatter asymmetry [13-16], it is interesting to establish the sufficient and necessary conditions for $C P$ conservation in the leptonic sector with massive Majorana neutrinos. Only when these conditions are spoiled in a specific model of neutrino masses can one explain the leptonic $C P$ violation and associate it with the dynamical generation of cosmological matter-antimatter asymmetry. This task has already been taken up in the literature, particularly by Branco et al. [17] in the language of the so-called weakbasis (WB) invariants [18]. The leptonic $C P$ violation in terms of WB invariants was discussed first by Pilaftsis in the context of resonant leptogenesis [19] and subsequently by several other authors $[20,21]$. The central idea of this approach is to define the general $C P$ transformation, which is actually a combination of the ordinary $C P$ transformation and the flavor-basis transformation. More explicitly, if the Lagrangian in Eq. (1.2) is invariant under the following transformations [17],

$$
\begin{array}{ll}
l_{\mathrm{L}} \rightarrow U_{\mathrm{L}} \mathcal{C} l_{\mathrm{L}}^{*}, & \nu_{\mathrm{L}} \rightarrow U_{\mathrm{L}} \mathcal{C} \nu_{\mathrm{L}}^{*}, \\
l_{\mathrm{R}} \rightarrow U_{\mathrm{R}} \mathcal{C} l_{\mathrm{R}}^{*}, & W_{\mu}^{-} \rightarrow-(-1)^{\delta_{0 \mu} W_{\mu}^{+},}
\end{array}
$$

where the asterisk indicates the complex conjugation and $\delta_{0 \mu}$ (for $\mu=0,1,2,3$ ) stands for the Kronecker delta, while $U_{\mathrm{L}}$ and $U_{\mathrm{R}}$ are two arbitrary $3 \times 3$ unitary matrices in the flavor space, then the sufficient and necessary conditions for $C P$ conservation are equivalent to the existence of two unitary matrices $U_{\mathrm{L}}$ and $U_{\mathrm{R}}$ such that the identities [17]

$$
U_{\mathrm{L}}^{\dagger} M_{\nu} U_{\mathrm{L}}^{*}=-M_{\nu}^{*}, \quad U_{\mathrm{L}}^{\dagger} M_{l} U_{\mathrm{R}}=M_{l}^{*}
$$

are satisfied. With the help of Eq. (1.4), one can find out the minimal set of sufficient and necessary conditions for $C P$ conservation in the leptonic sector in terms of WB invariants [22]

$$
\begin{gathered}
\mathcal{I}_{1} \equiv \operatorname{Tr}\left\{\left[H_{\nu}, H_{l}\right]^{3}\right\}=0, \\
\mathcal{I}_{2} \equiv \operatorname{Im}\left\{\operatorname{Tr}\left[H_{l} H_{\nu} G_{l \nu}\right]\right\}=0, \\
\mathcal{I}_{3} \equiv \operatorname{Tr}\left\{\left[G_{l \nu}, H_{l}\right]^{3}\right\}=0,
\end{gathered}
$$

where $H_{l} \equiv M_{l} M_{l}^{\dagger}, \quad H_{\nu} \equiv M_{\nu} M_{\nu}^{\dagger}$, and $G_{l \nu} \equiv M_{\nu} H_{l}^{*} M_{\nu}^{\dagger}$ have been introduced.

It was pointed out in Ref. [23] that those conditions in Eqs. (1.5)-(1.7) are not sufficient to guarantee $C P$ conservation in general. A numerical counterexample was given therein to illustrate that $C P$ violation still exists even when all three conditions in Eqs. (1.5)-(1.7) are satisfied. For this reason, a new set of three invariants $\left\{\mathcal{I}_{1}, \mathcal{I}_{2}, \mathcal{I}_{4}\right\}$ was suggested in Ref. [23], with $\mathcal{I}_{4}$ defined as

$$
\mathcal{I}_{4} \equiv \operatorname{Im}\left\{\operatorname{Tr}\left[H_{l} H_{\nu}^{2} G_{l \nu}\right]\right\}=0,
$$

which can guarantee $C P$ conservation at least in the experimentally allowed parameter space of lepton masses and mixing angles. ${ }^{1}$ Note that the invariance under the general $C P$ transformations in Eq. (1.3) requires $U_{\mathrm{L}}^{\dagger} H_{l} U_{\mathrm{L}}=$ $H_{l}^{*}, \quad U_{\mathrm{L}}^{\dagger} H_{\nu} U_{\mathrm{L}}=H_{\nu}^{*}$, and $U_{\mathrm{L}}^{\dagger} G_{l \nu} U_{\mathrm{L}}=G_{l \nu}^{*}$ according to Eq. (1.4). By using these transformation rules, one can immediately prove that $\mathcal{I}_{i}$ (for $i=1,2,3,4$ ) are indeed WB invariants. Given the lepton mass matrices $M_{l}$ and $M_{\nu}$ in a concrete model, the advantage of these WB invariants is to remove the ambiguity of flavor-basis transformations in judging whether $C P$ conservation is present.

In this work, we aim to derive the sufficient and necessary conditions for $C P$ conservation in the leptonic sector, and especially focus on the scenario of a partially or completely degenerate neutrino mass spectrum [24,25]. The number of flavor mixing angles and $C P$-violating phases in these special cases will be clarified. In addition, we investigate the radiative corrections to leptonic $C P$ violation by using the renormalization-group equations of the WB invariants. On the other hand, since neutrino oscillation experiments indicate that any two of three light neutrinos cannot be exactly degenerate in mass, we consider the mass degeneracy for heavy Majorana neutrinos in the canonical seesaw models $[20,26]$ and explore the implications of such a partial or complete mass degeneracy for the leptonic $C P$ violation at low- and high-energy scales.

The remaining parts of our paper are structured as follows. In Sec. II, we recall the sufficient and necessary conditions for the $C P$ violation in the low-energy effective theory of lepton masses and flavor mixing, and we pay particular attention to the cases of a partial or complete degeneracy in neutrino masses. The renormalization-group equations of the WB invariants will be derived and used to study the running behaviors of $C P$ phases. Then, we apply the formalism for light Majorana neutrinos to the case of heavy Majorana neutrinos in Sec. III. The full set of WB

\footnotetext{
${ }^{1}$ Here the number $n$ of vanishing WB invariants $\mathcal{I}_{1}=\mathcal{I}_{2}=$ $\mathcal{I}_{4}=0$ to guarantee $C P$ conservation coincides with the number of $C P$ phases in the theory, i.e., $n=3$ in the present case. However, this is valid under the assumption that the lepton masses and the flavor mixing angles do not take any contrived values. More explicitly, we assume that all the other physical parameters take the values within their experimentally allowed parameter space. If this is not assumed, as pointed out in Ref. [23], then there may still be $C P$ violation in a theory with $n C P$ phases, even when the properly chosen $n$ WB invariants vanish.
} 
invariants for $C P$ conservation will be given and utilized to analyze the possible connection between $C P$ violation at low- and high-energy scales. Finally, we summarize our main conclusions in Sec. IV.

\section{LOW-ENERGY EFFECTIVE THEORY}

At the low-energy scale, leptonic $C P$ violation arises from the complex mass matrices of charged leptons and light Majorana neutrinos, as indicated in Eq. (1.2). If three Majorana neutrinos are not degenerate in mass, the minimal set of sufficient and necessary conditions for $C P$ conservation have already been given in Eqs. (1.5), (1.6), and (1.8). Although the details can be found in Ref. [23], we briefly summarize the key points concerning these sufficient and necessary conditions for $C P$ conservation in the case of nondegenerate neutrino masses in order to establish our notation. As the WB invariants are by definition independent of basis transformations in the flavor space, it should be kept in mind that one can calculate them in any convenient basis and the final results depend only on physical parameters.

In the mass basis of charged leptons and light Majorana neutrinos, the $C P$-violating phases are contained in the PMNS matrix [1], which is usually parametrized in terms of three mixing angles $\left\{\theta_{12}, \theta_{13}, \theta_{23}\right\}$, one Dirac-type $C P$ phase $\delta$, and two Majorana-type $C P$ phases $\{\rho, \sigma\}$, namely,

$$
U=\left(\begin{array}{ccc}
c_{13} c_{12} & c_{13} s_{12} & s_{13} e^{-\mathrm{i} \delta} \\
-s_{12} c_{23}-c_{12} s_{13} s_{23} e^{\mathrm{i} \delta} & +c_{12} c_{23}-s_{12} s_{13} s_{23} e^{\mathrm{i} \delta} & c_{13} s_{23} \\
+s_{12} s_{23}-c_{12} s_{13} c_{23} e^{\mathrm{i} \delta} & -c_{12} s_{23}-s_{12} s_{13} c_{23} e^{\mathrm{i} \delta} & c_{13} c_{23}
\end{array}\right) \cdot\left(\begin{array}{ccc}
e^{\mathrm{i} \rho} & 0 & 0 \\
0 & e^{\mathrm{i} \sigma} & 0 \\
0 & 0 & 1
\end{array}\right)
$$

where $c_{i j} \equiv \cos \theta_{i j}$ and $s_{i j} \equiv \sin \theta_{i j}$ (for $i j=12,13,23$ ) have been defined. Therefore, the invariants $\left\{\mathcal{I}_{1}, \mathcal{I}_{2}, \mathcal{I}_{4}\right\}$ can be expressed in terms of the charged-lepton masses $\left\{m_{e}, m_{\mu}, m_{\tau}\right\}$, neutrino masses $\left\{m_{1}, m_{2}, m_{3}\right\}$, leptonic flavor mixing angles $\left\{\theta_{12}, \theta_{13}, \theta_{23}\right\}$, and $C P$-violating phases $\{\delta, \rho, \sigma\}$. It is straightforward to verify that the invariant $\mathcal{I}_{1}$ in Eq. (1.5) can be written as

$$
\mathcal{I}_{1}=-6 \mathrm{i} \Delta_{21} \Delta_{31} \Delta_{32} \Delta_{e \mu} \Delta_{\mu \tau} \Delta_{\tau e} \mathcal{J}
$$

where $\Delta_{i j} \equiv m_{i}^{2}-m_{j}^{2}$ (for $i, j=1,2,3$ ) are neutrino masssquared differences, $\Delta_{\alpha \beta} \equiv m_{\alpha}^{2}-m_{\beta}^{2}$ (for $\alpha, \beta=e, \mu, \tau$ ) are charged-lepton mass-squared differences, and $\mathcal{J} \equiv$ $\operatorname{Im}\left[U_{e 1} U_{e 2}^{*} U_{\mu 1}^{*} U_{\mu 2}\right]$ is the Jarlskog invariant for $C P$ violation in the leptonic sector [27-29]. For the standard parametrization of $U$ in Eq. (2.1), one can get the explicit expression $\mathcal{J}=s_{12} c_{12} s_{23} c_{23} s_{13} c_{13}^{2} \sin \delta$. In a similar way, the other two WB invariants $\mathcal{I}_{2}$ and $\mathcal{I}_{4}$ can also be calculated, but the explicit analytical expressions are too lengthy to list here. Taking the advantage of the simple result for $\mathcal{I}_{1}$ in Eq. (2.2), we can show that $\mathcal{I}_{1}=\mathcal{I}_{2}=$ $\mathcal{I}_{4}=0$ are sufficient conditions for $C P$ conservation in the case of nondegenerate neutrino masses. First of all, $\mathcal{I}_{1}=0$ holds if and only if $\delta=0$ or $\pi$. After using $\mathcal{I}_{1}=0$ to eliminate the Dirac $C P$ phase $\delta$, we can then observe that $\mathcal{I}_{2}=0$ and $\mathcal{I}_{4}=0$ give rise to two independent equations of two Majorana $C P$ phases $\rho$ and $\sigma$, namely,

$$
\begin{aligned}
& f_{1} \sin (2 \rho)+f_{2} \sin (2 \sigma)+f_{3} \sin (2 \rho-2 \sigma)=0, \\
& h_{1} \sin (2 \rho)+h_{2} \sin (2 \sigma)+h_{3} \sin (2 \rho-2 \sigma)=0,
\end{aligned}
$$

where $f_{i}$ and $h_{i}$ (for $i=1,2,3$ ) are functions of three mixing angles and six lepton masses. The explicit expressions of $f_{i}$ and $h_{i}$ can be found in Ref. [23]. Since Eqs. (2.3) and (2.4) are actually nonlinear in nature, they cannot force $\rho$ and $\sigma$ to take only trivial values (i.e., 0 or $\pi / 2$ ) in general. However, it can be proved that at least in the whole physically allowed parameter space, these two equations are sufficient to ensure that $\rho$ and $\sigma$ take only trivial values [23], so $C P$ conservation is justified. On the other hand, it is easy to prove that the vanishing of three invariants $\left\{\mathcal{I}_{1}, \mathcal{I}_{2}, \mathcal{I}_{4}\right\}$ is also a necessary condition for $C P$ conservation with nondegenerate neutrino masses [17].

In the following discussions, we shall concentrate on the partially degenerate mass spectrum ${ }^{2} m_{1}=m_{2} \neq m_{3}$ and the completely degenerate mass spectrum $m_{1}=$ $m_{2}=m_{3}$. These two special cases were not considered in Ref. [23].

\section{A. Partial mass degeneracy}

If the partial mass degeneracy $m_{1}=m_{2} \neq m_{3}$ is assumed, then from Eq. (2.2) we find that $\mathcal{I}_{1}$ vanishes automatically and it can no longer be used to investigate the properties of $C P$ violation. Nevertheless, there exists an extra degree of freedom in the system with two degenerate neutrino masses, which can be implemented to reduce the number of $C P$-violating phases.

To see this point more clearly, we choose the basis where both neutrino mass matrix $M_{\nu}$ and the chargedcurrent interaction are flavor diagonal. In this basis, the neutrino mass matrix $M_{\nu}=\hat{M}_{\nu}=\operatorname{diag}\left\{m, m, m_{3}\right\}$, where we have taken $m_{1}=m_{2}=m$, is invariant under the transformation

\footnotetext{
${ }^{2}$ The other two possibilities, i.e., $m_{1} \neq m_{2}=m_{3}$ and $m_{1}=m_{3} \neq m_{2}$, can be examined in a similar way.
} 


$$
\begin{aligned}
\left(\begin{array}{l}
\nu_{1 \mathrm{~L}} \\
\nu_{2 \mathrm{~L}} \\
\nu_{3 \mathrm{~L}}
\end{array}\right) & \rightarrow\left(\begin{array}{c}
\nu_{1 \mathrm{~L}}^{\prime} \\
\nu_{2 \mathrm{~L}}^{\prime} \\
\nu_{3 \mathrm{~L}}^{\prime}
\end{array}\right)=R_{12}^{\dagger}(\alpha)\left(\begin{array}{l}
\nu_{1 \mathrm{~L}} \\
\nu_{2 \mathrm{~L}} \\
\nu_{3 \mathrm{~L}}
\end{array}\right) \\
& \equiv\left(\begin{array}{ccc}
\cos \alpha & -\sin \alpha & 0 \\
\sin \alpha & \cos \alpha & 0 \\
0 & 0 & 1
\end{array}\right)\left(\begin{array}{c}
\nu_{1 \mathrm{~L}} \\
\nu_{2 \mathrm{~L}} \\
\nu_{3 \mathrm{~L}}
\end{array}\right),
\end{aligned}
$$

where $R_{12}(\alpha)$ has been defined as the rotation matrix in the $(1,2)$ plane, with $\alpha$ being an arbitrary real rotation angle. To keep the flavor-diagonal charged-current interaction unchanged, one thus has to make the same transformation on the left-handed charged leptons simultaneously, i.e.,

$$
\begin{aligned}
\left(\begin{array}{c}
e_{\mathrm{L}} \\
\mu_{\mathrm{L}} \\
\tau_{\mathrm{L}}
\end{array}\right) & \rightarrow\left(\begin{array}{c}
e_{\mathrm{L}}^{\prime} \\
\mu_{\mathrm{L}}^{\prime} \\
\tau_{\mathrm{L}}^{\prime}
\end{array}\right)=R_{12}^{\dagger}(\alpha)\left(\begin{array}{c}
e_{\mathrm{L}} \\
\mu_{\mathrm{L}} \\
\tau_{\mathrm{L}}
\end{array}\right) \\
& \equiv\left(\begin{array}{ccc}
\cos \alpha & -\sin \alpha & 0 \\
\sin \alpha & \cos \alpha & 0 \\
0 & 0 & 1
\end{array}\right)\left(\begin{array}{l}
e_{\mathrm{L}} \\
\mu_{\mathrm{L}} \\
\tau_{\mathrm{L}}
\end{array}\right) .
\end{aligned}
$$

Under these transformations, the entire effective Lagrangian in Eq. (1.2) is not modified except for the charged-lepton mass matrix $M_{l}$, which together with $H_{l} \equiv$ $M_{l} M_{l}^{\dagger}$ transforms as follows:

$$
\begin{aligned}
M_{l} \rightarrow M_{l}^{\prime} & =R_{12}^{\dagger}(\alpha) M_{l}, \\
H_{l} \rightarrow H_{l}^{\prime} & =R_{12}^{\dagger}(\alpha) H_{l} R_{12}(\alpha) .
\end{aligned}
$$

In the chosen basis, only the charged-lepton mass matrix $M_{l}$ is complex, and thus it contains all the information about $C P$-violating phases. Furthermore, to remove the unphysical phases related to the right-handed chargedlepton fields, we consider only the Hermitian matrix $H_{l}$. Now we show that the rotation matrix $R_{12}(\alpha)$ can be utilized to reduce the number of $C P$ phases in the original Lagrangian. To be explicit, we directly establish the correspondence between the matrix elements of $H_{l}^{\prime}$ and those of $H_{l}$, i.e.,

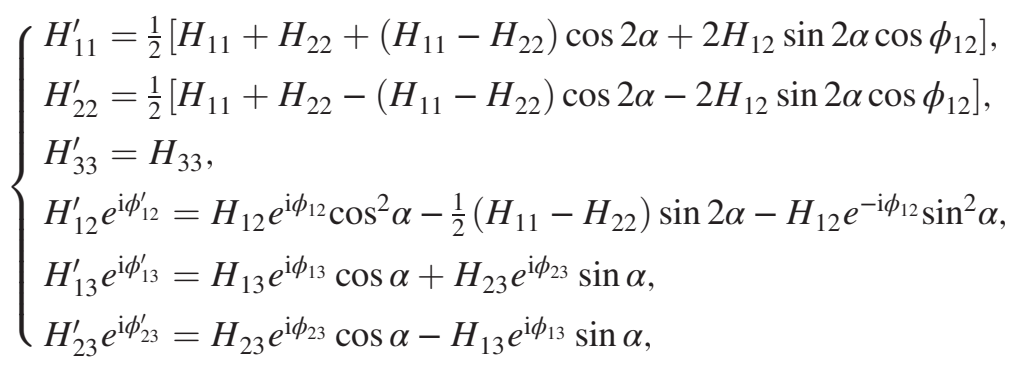

where $H_{i j} \equiv\left|\left(H_{l}\right)_{i j}\right|$ and $\phi_{i j} \equiv \arg \left[\left(H_{l}\right)_{i j}\right]$ have been defined for $H_{l}$ (for $i, j=1,2,3$ ), and likewise $H_{i j}^{\prime} \equiv$ $\left|\left(H_{l}^{\prime}\right)_{i j}\right|$ and $\phi_{i j}^{\prime} \equiv \arg \left[\left(H_{l}^{\prime}\right)_{i j}\right]$ for $H_{l}^{\prime}$. Note that $H_{l}$ (or $\left.H_{l}^{\prime}\right)$ is Hermitian, so only three phases $\left\{\phi_{12}, \phi_{13}, \phi_{23}\right\}$ in $H_{l}$ (or $\left\{\phi_{12}^{\prime}, \phi_{13}^{\prime}, \phi_{23}^{\prime}\right\}$ in $H_{l}^{\prime}$ ) are independent. In the case of nondegenerate neutrino masses, where these three phases are all physical, three conditions $\mathcal{I}_{1}=0, \mathcal{I}_{2}=0$, and $\mathcal{I}_{4}=0$ are needed to guarantee $C P$ conservation. In the presence of mass degeneracy $m_{1}=m_{2}$, we can adjust the rotation angle $\alpha$ to eliminate one phase in $H_{l}^{\prime}$. For example, if we set

$$
\tan \alpha=-\frac{H_{13} \sin \phi_{13}}{H_{23} \sin \phi_{23}},
$$

then one can immediately verify that $\phi_{13}^{\prime}=0$ holds or equivalently that $\left(H_{l}^{\prime}\right)_{13}$ is real, with the help of Eq. (2.9). This is true for the most general case of $H_{23} \sin \phi_{23} \neq 0$. In the special case of $H_{23} \sin \phi_{23}=0$, we can observe from Eq. (2.9) that $H_{23}^{\prime} \sin \phi_{23}^{\prime}=-H_{13} \sin \phi_{13} \sin \alpha$ and $H_{13}^{\prime} \sin \phi_{13}^{\prime}=H_{13} \sin \phi_{13} \cos \alpha$, so it is possible to eliminate $\phi_{23}^{\prime}$ or $\phi_{13}^{\prime}$ by setting $\alpha=0$ or $\pi / 2$.

In general, we are left with only two phases $\left\{\phi_{12}^{\prime}, \phi_{23}^{\prime}\right\}$ in $H_{l}^{\prime}$, while $M_{\nu}^{\prime}$ is real and diagonal. Therefore, we can prove that only two WB invariants are needed to ensure $C P$ conservation in the leptonic sector, which will be taken to be $\left\{\mathcal{I}_{2}, \mathcal{I}_{3}\right\}$. It is worth stressing that the choice of two independent WB invariants is by no means unique, and $\left\{\mathcal{I}_{2}, \mathcal{I}_{3}\right\}$ are chosen simply for illustration. The proof is as follows.

(a) Now that $M_{\nu}^{\prime}=\operatorname{diag}\left\{m, m, m_{3}\right\}$ is real and diagonal, we can directly compute the WB invariant $\mathcal{I}_{2}$ in Eq. (1.6) with $\phi_{13}^{\prime}=0$ in $H_{l}^{\prime}$. The analytical expression turns out to be quite simple, namely,

$$
\mathcal{I}_{2}=m m_{3}\left(m_{3}^{2}-m^{2}\right) H_{23}^{\prime 2} \sin 2 \phi_{23}^{\prime},
$$

so $\mathcal{I}_{2}=0$ leads to $\phi_{23}^{\prime}=0$ or $\phi_{23}^{\prime}=\pi / 2$. In both cases, one can find that the WB invariant $\mathcal{I}_{3}$ depends 
on the phase $\phi_{12}^{\prime}$. More explicitly, for $\phi_{23}^{\prime}=0$, we have $\mathcal{I}_{3} \propto \sin \phi_{12}^{\prime}$, while for $\phi_{23}^{\prime}=\pi / 2$ we get $\mathcal{I}_{3} \propto \cos \phi_{12}^{\prime}$. As a consequence, together with $\mathcal{I}_{3}=0, \mathcal{I}_{2}=0$ implies that $\left\{\phi_{12}^{\prime}=0, \phi_{23}^{\prime}=0, \phi_{13}^{\prime}=0\right\}$ or $\left\{\phi_{12}^{\prime}=\pi / 2, \phi_{23}^{\prime}=\pi / 2, \phi_{13}^{\prime}=0\right\}$. In either case, these trivial phases are expected for the absence of $C P$ violation. (b) On the other hand, one can relate the $C P$-violating phases in $H_{l}^{\prime}$ to those in the PMNS matrix $U$. In the chosen basis, we have $M_{l}^{\prime}=U^{\dagger} \hat{M}_{l}$ and $H_{l}^{\prime}=U^{\dagger} \hat{D}_{l} U$, where $\hat{M}_{l} \equiv \operatorname{diag}\left\{m_{e}, m_{\mu}, m_{\tau}\right\}$ and $\hat{D}_{l} \equiv \widehat{M_{l}^{2}}=\operatorname{diag}\left\{m_{e}^{2}\right.$, $\left.m_{\mu}^{2}, m_{\tau}^{2}\right\}$. Then it is possible to relate the three phases in $H_{l}^{\prime}$ to the three physical phases in the PMNS matrix,

$$
\begin{aligned}
H_{12}^{\prime} e^{\mathrm{i} \phi_{12}^{\prime}}= & {\left[\left(s_{12}^{2} e^{\mathrm{i} \delta}-c_{12}^{2} e^{-\mathrm{i} \delta}\right) s_{13} s_{23} c_{23}+s_{12} c_{12}\left(s_{23}^{2}-c_{23}^{2}\right)\right] \Delta_{\mu \tau} e^{-\mathrm{i}(\rho-\sigma)} } \\
& +\left(\Delta_{e \mu} s_{23}^{2}-\Delta_{\tau e} c_{23}^{2}\right) s_{12} c_{12} c_{13}^{2} e^{-\mathrm{i}(\rho-\sigma)}, \\
H_{13}^{\prime} e^{\mathrm{i} \phi_{13}^{\prime}=} & \left(\Delta_{e \mu} s_{23}^{2}-\Delta_{\tau e} c_{23}^{2}\right) c_{12} s_{13} c_{13} e^{-\mathrm{i}(\rho+\delta)}-\Delta_{\mu \tau} s_{12} c_{13} s_{23} c_{23} e^{-\mathrm{i} \rho}, \\
H_{23}^{\prime} e^{\mathrm{i} \phi_{23}^{\prime}}= & \left(\Delta_{e \mu} s_{23}^{2}-\Delta_{\tau e} c_{23}^{2}\right) s_{12} s_{13} c_{13} e^{-\mathrm{i}(\sigma+\delta)}+\Delta_{\mu \tau} c_{12} c_{13} s_{23} c_{23} e^{-\mathrm{i} \sigma},
\end{aligned}
$$

where it is interesting to observe that the expression of $H_{23}^{\prime} e^{\mathrm{i} \phi_{23}^{\prime}}$ can be obtained from that of $H_{13}^{\prime} e^{\mathrm{i} \phi_{13}^{\prime}}$ by simply replacing $\theta_{12}$ with $\theta_{12}-\pi / 2$ and $\rho$ with $\sigma$. Then $\left\{\phi_{12}^{\prime}=\right.$ $\left.\phi_{13}^{\prime}=\phi_{23}^{\prime}=0\right\}$ or $\left\{\phi_{12}^{\prime}=\phi_{23}^{\prime}=\pi / 2, \phi_{13}^{\prime}=0\right\}$ is equivalent to $\{\delta=\rho=\sigma=0\}$ or $\{\delta=\rho=0, \sigma=\pi / 2\}$, which is equivalent to $C P$ conservation. ${ }^{3}$ This completes the proof that $\left\{\mathcal{I}_{2}=0, \mathcal{I}_{3}=0\right\}$ constitute the sufficient and necessary conditions of $C P$ conservation in the case of partial mass degeneracy.

In summary, for the partial degeneracy of neutrino masses $m_{1}=m_{2} \neq m_{3}$, there are only two independent $C P$-violating phases, and the vanishing of two WB invariants in Eqs. (1.6) and (1.7), namely, $\mathcal{I}_{2}=0$ and $\mathcal{I}_{3}=0$, serves as the sufficient and necessary condition for the leptonic $C P$ conservation. In addition, it is worthwhile to notice that the freedom associated with the mass degeneracy $m_{1}=m_{2}$ can be implemented to reduce the number of $C P$-violating phases by one, leaving three flavor mixing angles intact.

\section{B. Complete mass degeneracy}

If neutrino masses are completely degenerate, i.e., $m_{1}=m_{2}=m_{3} \equiv m$, then it is straightforward to verify that the WB invariants $\mathcal{I}_{1}, \mathcal{I}_{2}$, and $\mathcal{I}_{4}$ automatically vanish, whereas $\mathcal{I}_{3}$ is generally nonzero. However, compared to the case of partial mass degeneracy, the complete mass degeneracy allows for more degrees of freedom, which can be utilized to reduce the number of physical $C P$ violating phases.

In the same way as for the partial mass degeneracy, working in the basis where the neutrino mass matrix

\footnotetext{
${ }^{3}$ It should be noted that $\rho$ and $\sigma$ are Majorana-type $C P$ violating phases and that the $C P$ symmetry is still conserved when they take the value of $\pi / 2$ in the standard parametrization in Eq. (2.1). The properties of three phases in $H_{l}$ are quite different. For instance, if one of three phases in $H_{l}$ takes the value of $\pi / 2$ and the other two are zero, then the $C P$ symmetry is violated.
}

$M_{\nu}=\hat{M}_{\nu}=\operatorname{diag}\{m, m, m\}$ is real and diagonal, we can introduce two successive rotations in the flavor basis

$$
\begin{gathered}
\left(\begin{array}{c}
\nu_{1 \mathrm{~L}} \\
\nu_{2 \mathrm{~L}} \\
\nu_{3 \mathrm{~L}}
\end{array}\right) \rightarrow\left(\begin{array}{c}
\nu_{1 \mathrm{~L}}^{\prime} \\
\nu_{2 \mathrm{~L}}^{\prime} \\
\nu_{3 \mathrm{~L}}^{\prime}
\end{array}\right)=\left[R_{12}(\alpha) R_{13}(\beta)\right]^{\dagger}\left(\begin{array}{c}
\nu_{1 \mathrm{~L}} \\
\nu_{2 \mathrm{~L}} \\
\nu_{3 \mathrm{~L}}
\end{array}\right), \\
\left(\begin{array}{c}
e_{\mathrm{L}} \\
\mu_{\mathrm{L}} \\
\tau_{\mathrm{L}}
\end{array}\right) \rightarrow\left(\begin{array}{c}
e_{\mathrm{L}}^{\prime} \\
\mu_{\mathrm{L}}^{\prime} \\
\tau_{\mathrm{L}}^{\prime}
\end{array}\right)=\left[R_{12}(\alpha) R_{13}(\beta)\right]^{\dagger}\left(\begin{array}{c}
e_{\mathrm{L}} \\
\mu_{\mathrm{L}} \\
\tau_{\mathrm{L}}
\end{array}\right),
\end{gathered}
$$

where the rotation matrices are defined as

$$
\begin{aligned}
& R_{12}(\alpha)=\left(\begin{array}{ccc}
\cos \alpha & -\sin \alpha & 0 \\
\sin \alpha & \cos \alpha & 0 \\
0 & 0 & 1
\end{array}\right), \\
& R_{13}(\beta)=\left(\begin{array}{ccc}
\cos \beta & 0 & \sin \beta \\
0 & 1 & 0 \\
-\sin \beta & 0 & \cos \beta
\end{array}\right),
\end{aligned}
$$

with $\alpha$ and $\beta$ being two arbitrary real rotation angles. After these rotations, the neutrino mass matrix $M_{\nu}$ is unchanged and the charged-current interaction remains flavor diagonal, but the charged-lepton mass matrix $H_{l} \equiv$ $M_{l} M_{l}^{\dagger}$ transforms as

$H_{l} \rightarrow H_{l}^{\prime}=\left[R_{12}(\alpha) R_{13}(\beta)\right]^{\dagger} \cdot H_{l} \cdot\left[R_{12}(\alpha) R_{13}(\beta)\right]$,

which contains all the physical $C P$-violating phases.

Similar to what we have done in Sec. II A, we can show how to adjust $\alpha$ and $\beta$ to eliminate two $C P$-violating phases in $H_{l}^{\prime}$. This is equivalent to the reduction of the total number of $C P$-violating phases in the leptonic sector by two. After some straightforward calculations, we find that if $\alpha$ and $\beta$ are taken to be 
$\tan \alpha=-\frac{H_{13} \sin \phi_{13}}{H_{23} \sin \phi_{23}}, \quad \tan \beta=\frac{H_{23} \sin \phi_{23}}{H_{12} \sin \phi_{12}} \frac{1}{\cos \alpha}$,

then $\sin \phi_{13}^{\prime}=\sin \phi_{23}^{\prime}=0$, indicating that the imaginary parts of the matrix elements $\left(H_{l}^{\prime}\right)_{13}$ and $\left(H_{l}^{\prime}\right)_{23}$ vanish. As a result, one needs only one vanishing WB invariant, e.g., $\mathcal{I}_{3}=0$, to eliminate the remaining one $C P$-violating phase in $H_{l}^{\prime}$. After setting $\phi_{13}^{\prime}=\phi_{23}^{\prime}=0$ or $\pi$, we can greatly simplify the explicit expression of $\mathcal{I}_{3}$, namely,

$$
\begin{aligned}
\mathcal{I}_{3}= & -48 \mathrm{i} H_{12}^{\prime 3} m^{6}\left[H_{13}^{\prime} H_{23}^{\prime}\left(H_{22}^{\prime}-H_{11}^{\prime}\right)\right. \\
& \left.+H_{12}^{\prime}\left(H_{13}^{\prime 2}-H_{23}^{\prime 2}\right) \cos \phi_{12}^{\prime}\right] \sin ^{3} \phi_{12}^{\prime},
\end{aligned}
$$

implying that $\mathcal{I}_{3}=0$ gives rise to $\phi_{12}^{\prime}=0$ or $\pi$ if the whole coefficient in front of $\sin ^{3} \phi_{12}^{\prime}$ is not fine-tuned to be zero. Therefore, $\mathcal{I}_{3}=0$ is the sufficient and necessary condition for $C P$ conservation in the case of complete neutrino mass degeneracy, which is consistent with the conclusion previously drawn in Ref. [24].

Generally speaking, the neutrino mass matrix $M_{\nu}=$ $\hat{M}_{\nu} \equiv \operatorname{diag}\{m, m, m\}$ in the case of complete mass degeneracy is invariant under an arbitrary orthogonal rotation with three rotation angles. One may wonder whether it is possible to eliminate all three $C P$-violating phases. Now we demonstrate that this is impossible. To this end, we first carry out the most general orthogonal rotation in the flavor basis

$$
\begin{aligned}
& \left(\begin{array}{c}
\nu_{1 \mathrm{~L}} \\
\nu_{2 \mathrm{~L}} \\
\nu_{3 \mathrm{~L}}
\end{array}\right) \rightarrow\left(\begin{array}{c}
\nu_{1 \mathrm{~L}}^{\prime} \\
\nu_{2 \mathrm{~L}}^{\prime} \\
\nu_{3 \mathrm{~L}}^{\prime}
\end{array}\right)=\left[R_{12}(\alpha) R_{13}(\beta) R_{23}(\gamma)\right]^{\dagger}\left(\begin{array}{c}
\nu_{1 \mathrm{~L}} \\
\nu_{2 \mathrm{~L}} \\
\nu_{3 \mathrm{~L}}
\end{array}\right), \\
& \left(\begin{array}{c}
e_{\mathrm{L}} \\
\mu_{\mathrm{L}} \\
\tau_{\mathrm{L}}
\end{array}\right) \rightarrow\left(\begin{array}{c}
e_{\mathrm{L}}^{\prime} \\
\mu_{\mathrm{L}}^{\prime} \\
\tau_{\mathrm{L}}^{\prime}
\end{array}\right)=\left[R_{12}(\alpha) R_{13}(\beta) R_{23}(\gamma)\right]^{\dagger}\left(\begin{array}{c}
e_{\mathrm{L}} \\
\mu_{\mathrm{L}} \\
\tau_{\mathrm{L}}
\end{array}\right)
\end{aligned}
$$

where $R_{12}(\alpha)$ and $R_{13}(\beta)$ are the same as before and

$$
R_{23}(\gamma)=\left(\begin{array}{ccc}
1 & 0 & 0 \\
0 & \cos \gamma & -\sin \gamma \\
0 & \sin \gamma & \cos \gamma
\end{array}\right),
$$

with $\gamma$ being another real arbitrary rotation angle. Such transformations will keep the neutrino mass matrix and the charged-current interaction unchanged. However, it is straightforward to prove that the third degree of freedom can be used to eliminate only a flavor mixing angle rather than the remaining $C P$-violating phase. One can accomplish the proof by contradiction. First, given Eq. (2.17), the Hermitian matrix $H_{l}$ transforms as

$$
\begin{aligned}
H_{l} & \rightarrow H_{l}^{\prime} \\
& =\left[R_{12}(\alpha) R_{13}(\beta) R_{23}(\gamma)\right]^{\dagger} \cdot H_{l} \cdot\left[R_{12}(\alpha) R_{13}(\beta) R_{23}(\gamma)\right],
\end{aligned}
$$

and we suppose that all three $\mathrm{CP}$-violating phases in $H_{l}^{\prime}$ can be made trivial (i.e., $\phi_{12}^{\prime}, \phi_{23}^{\prime}, \phi_{13}^{\prime}=0$ or $\pi$ ) by adjusting the rotation angles $\alpha, \beta$, and $\gamma$. If this is possible, then we can observe that the imaginary parts of three off-diagonal elements of $H_{l}^{\prime}$ in Eq. (2.18) should vanish, i.e.,

$$
\begin{aligned}
\left(\begin{array}{l}
H_{12}^{\prime} \sin \phi_{12}^{\prime} \\
H_{23}^{\prime} \sin \phi_{23}^{\prime} \\
H_{13}^{\prime} \sin \phi_{13}^{\prime}
\end{array}\right)= & \left(\begin{array}{ccc}
c_{\beta} c_{\gamma} & s_{\alpha} s_{\gamma}+c_{\alpha} s_{\beta} c_{\gamma} & c_{\alpha} s_{\gamma}-s_{\alpha} s_{\beta} c_{\gamma} \\
-c_{\beta} s_{\gamma} & s_{\alpha} c_{\gamma}-c_{\alpha} s_{\beta} s_{\gamma} & c_{\alpha} c_{\gamma}+s_{\alpha} s_{\beta} s_{\gamma} \\
-s_{\beta} & c_{\alpha} c_{\beta} & -s_{\alpha} c_{\beta}
\end{array}\right) \\
& \times\left(\begin{array}{c}
H_{12} \sin \phi_{12} \\
H_{23} \sin \phi_{23} \\
H_{13} \sin \phi_{13}
\end{array}\right)=\mathbf{0}
\end{aligned}
$$

which is a system of homogeneous linear equations for $H_{12} \sin \phi_{12}, H_{23} \sin \phi_{23}$, and $H_{13} \sin \phi_{13}$. Note that $s_{\alpha} \equiv$ $\sin \alpha$ and $c_{\alpha} \equiv \cos \alpha$ were defined in Eq. (2.19), and likewise for $\beta$ and $\gamma$. It is interesting to notice that the determinant of the $3 \times 3$ coefficient matrix in the middle of Eq. (2.19) is actually -1 , which is independent of $\alpha, \beta$, and $\gamma$. Therefore, Eq. (2.19) holds if and only if $\sin \phi_{12}=\sin \phi_{23}=$ $\sin \phi_{13}=0$, which runs into a contradiction with the fact that there are in general three $\mathrm{CP}$-violating phases in $H_{l}$. So this proves that even in the limit of complete mass degeneracy, there is still one nonvanishing phase so that $C P$ can be violated in the leptonic sector, which is consistent with the conclusion drawn in Ref. [24].

To use the third degree of freedom to eliminate a flavor mixing angle, we can first choose the rotation angles $\alpha$ and $\beta$ to obtain $\phi_{23}^{\prime}=0$ and $\phi_{12}^{\prime}=\phi_{13}^{\prime}$, and then $H_{l}^{\prime}$ can be explicitly written as

$$
\begin{aligned}
H_{l}^{\prime} & =P_{l}\left(\begin{array}{lll}
H_{11}^{\prime} & H_{12}^{\prime} & H_{13}^{\prime} \\
H_{12}^{\prime} & H_{22}^{\prime} & H_{23}^{\prime} \\
H_{13}^{\prime} & H_{23}^{\prime} & H_{33}^{\prime}
\end{array}\right) P_{l}^{\dagger} \\
& =\left(P_{l} O_{l}\right) \cdot\left(\begin{array}{ccc}
m_{e}^{2} & 0 & 0 \\
0 & m_{\mu}^{2} & 0 \\
0 & 0 & m_{\tau}^{2}
\end{array}\right) \cdot\left(P_{l} O_{l}\right)^{\dagger},
\end{aligned}
$$

where $P_{l} \equiv \operatorname{diag}\left\{e^{\mathrm{i} \phi_{12}^{\prime}}, 1,1\right\}$ and $O_{l}$ is the $3 \times 3$ orthogonal matrix that can be used to diagonalize the real and symmetric matrix $P_{l}^{\dagger} H_{l}^{\prime} P_{l}$. Since the neutrino mass matrix is already diagonal, the PMNS matrix is simply given by $U=\left(P_{l} O_{l}\right)^{\dagger}=O_{l}^{\mathrm{T}} P_{l}^{*}$. Furthermore, noticing that the mass eigenstates $\nu_{2}$ and $\nu_{3}$ are now degenerate in mass and that their Majorana $C P$ phases are both vanishing, we are allowed to rotate away one mixing angle by choosing the 
particular parametrization of $O_{l}$, as explicitly shown in Ref. [25].

To summarize, in the case of complete mass degeneracy, we are left with one $C P$-violating phase and two mixing angles. This should be compared with the case of partial mass degeneracy, where two $C P$-violating phases and three mixing angles are retained. It is worth mentioning that Ref. [25] examines the case where both neutrino masses and the associated Majorana $C P$-violating phases are partially or completely degenerate at the same time, which is quite different from the scenario under consideration. It is physically inequivalent to assume the equality of two Majorana $C P$ phases before or after the elimination of one mixing angle.

\section{The massless limit}

Current neutrino oscillation data still permit the lightest neutrino to be massless, so we make a brief comment on this particular situation. Without loss of generality, we take $m_{1}=0$ in the case of normal neutrino mass ordering (i.e., $m_{1}<m_{2}<m_{3}$ ).

If $m_{1}=0$ holds, then the Majorana $C P$-violating phase $\rho$ associated with the mass eigenstate $\nu_{1}$ automatically disappears from the theory. In this case, we are left with two $C P$-violating phases and need to require two $\mathrm{WB}$ invariants to vanish in order to ensure $C P$ conservation. Luckily, none of the previously introduced four WB invariants $\mathcal{I}_{i}$ (for $i=1,2,3,4)$ vanish in the limit of $m_{1}=0$, so we can choose any two of them to guarantee $C P$ conservation. To be more concrete, we take the set $\left\{\mathcal{I}_{1}, \mathcal{I}_{2}\right\}$. First, we can use $\mathcal{I}_{1}=0$ to eliminate the Dirac $C P$-violating phase $\delta$, since $\mathcal{I}_{1}$ is proportional to $\sin \delta$. Now that both $\delta$ and $\rho$ are set at zero, $\mathcal{I}_{2}$ turns out to be proportional to $\sin 2 \sigma$, where $\sigma$ denotes the remaining Majorana $C P$-violating phase. Then, the condition $\mathcal{I}_{2}=0$ enforces $\sigma$ to take only trivial values (i.e., 0 or $\pi / 2$ ), thus implying $C P$ conservation.

Therefore, in the limit $m_{1}=0$, the vanishing of the set of two WB invariants $\left\{\mathcal{I}_{1}, \mathcal{I}_{2}\right\}$ serves as the sufficient and necessary condition for $C P$ conservation. As we shall see in the next section, the lightest neutrino is indeed massless at the tree level in the minimal seesaw model, which leads to the massless limit of the low-energy effective theory under consideration.

\section{Renormalization-group running}

In this section, we derive the renormalization-group equations (RGEs) of the WB invariants in leptonic sector in the effective theory, which have rarely been investigated in the literature. ${ }^{4}$ These RGEs can be applied to examine the evolution of the WB invariants and establish the connection between $C P$ violation at low- and high-energy

\footnotetext{
${ }^{4}$ The renormalization-group evolution of the WB invariants in the quark sector was discussed in Ref. [30].
}

scales. At the one-loop level, the evolution of the effective Majorana neutrino mass matrix $M_{\nu}$ and the chargedlepton mass matrix $M_{l}$ are governed by the following RGEs [31-43]:

$$
\begin{gathered}
\frac{\mathrm{d} M_{\nu}}{\mathrm{d} t}=\alpha_{\nu} M_{\nu}-\frac{3}{2}\left[\left(Y_{l} Y_{l}^{\dagger}\right) M_{\nu}+M_{\nu}\left(Y_{l} Y_{l}^{\dagger}\right)^{\mathrm{T}}\right], \\
\frac{\mathrm{d} M_{l}}{\mathrm{~d} t}=\alpha_{l} M_{l}+\frac{3}{2}\left(Y_{l} Y_{l}^{\dagger}\right) M_{l},
\end{gathered}
$$

where $t \equiv \ln \left(\mu / \Lambda_{\mathrm{EW}}\right) /\left(16 \pi^{2}\right)$ has been defined with $\Lambda_{\mathrm{EW}}$ being the electroweak scale and $\mu$ being the renormalization scale between $\Lambda_{\mathrm{EW}}$ and the seesaw scale. In the SM framework, we have $\alpha_{\nu} \approx-3 g_{2}^{2}+\lambda+6 y_{t}^{2}$ and $\alpha_{l} \approx$ $-9 g_{1}^{2} / 4-9 g_{2}^{2} / 4+3 y_{t}^{2}$, where $g_{1}$ and $g_{2}$ are the SM gauge couplings, $y_{t}$ is the top-quark Yukawa coupling, and $\lambda$ is the quartic Higgs coupling [41].

Starting with Eqs. (2.21) and (2.22) and recalling the definitions of $H_{l} \equiv M_{l} M_{l}^{\dagger}, H_{\nu} \equiv M_{\nu} M_{\nu}^{\dagger}$, and $G_{l \nu} \equiv M_{\nu} H_{l}^{*} M_{\nu}^{\dagger}$, one can easily find

$$
\frac{\mathrm{d} H_{l}}{\mathrm{~d} t}=2 \alpha_{l} H_{l}+6 H_{l}^{2} / v^{2}
$$

$$
\begin{aligned}
& \frac{\mathrm{d} H_{\nu}}{\mathrm{d} t}=2 \alpha_{\nu} H_{\nu}-3\left(H_{l} H_{\nu}+H_{\nu} H_{l}\right) / v^{2}-6 G_{l \nu} / v^{2}, \\
& \frac{\mathrm{d} G_{l \nu}}{\mathrm{d} t}=2\left(\alpha_{\nu}+\alpha_{l}\right) G_{l \nu}-3\left(G_{l \nu} H_{l}+H_{l} G_{l \nu}\right) / v^{2},
\end{aligned}
$$

where the relation $Y_{l}=\sqrt{2} M_{l} / v$ has been used. It is then straightforward to calculate the RGEs of the WB invariants $\mathcal{I}_{i}$ (for $\left.i=1,2,3,4\right)$. The final results are summarized as follows.

(a) First, as shown in Eq. (2.2), $\mathcal{I}_{1}$ is proportional to the Jarlskog invariant $\mathcal{J}$, which depends only on the Dirac $C P$-violating phase $\delta$ in the standard parametrization of the PMNS matrix. For this WB invariant, we have

$$
\begin{aligned}
\frac{\mathrm{d} \mathcal{I}_{1}}{\mathrm{~d} t} & =\operatorname{Tr}\left\{\frac{\mathrm{d}}{\mathrm{d} t}\left[H_{\nu}, H_{l}\right]^{3}\right\} \\
& =6\left(\alpha_{\nu}+\alpha_{l}\right) \mathcal{I}_{1}+9 \mathcal{I}_{1}^{(1)} / v^{2}-18 \mathcal{I}_{1}^{(2)} / v^{2},
\end{aligned}
$$

where $\mathcal{I}_{1}^{(1)} \equiv \operatorname{Tr}\left\{\left[H_{\nu}, H_{l}\right]^{2} \cdot\left[H_{\nu}, H_{l}^{2}\right]\right\} \quad$ and $\quad \mathcal{I}_{1}^{(2)} \equiv$ $\operatorname{Tr}\left\{\left[H_{\nu}, H_{l}\right]^{2} \cdot\left[G_{l \nu}, H_{l}\right]\right\}$ are also two WB invariants. Interestingly, it is easy to derive the explicit expression of $\mathcal{I}_{1}^{(1)}$, i.e.,

$$
\begin{aligned}
\mathcal{I}_{1}^{(1)} & =-4 \mathrm{i}\left(m_{e}^{2}+m_{\mu}^{2}+m_{\tau}^{2}\right) \Delta_{21} \Delta_{31} \Delta_{32} \Delta_{e \mu} \Delta_{\mu \tau} \Delta_{\tau e} \mathcal{J} \\
& =\frac{2}{3}\left(m_{e}^{2}+m_{\mu}^{2}+m_{\tau}^{2}\right) \mathcal{I}_{1},
\end{aligned}
$$


which is proportional to $\mathcal{I}_{1}$ itself and thus to the Jarlskog invariant $\mathcal{J}$. In the derivation of Eq. (2.27), we have made use of the identities $\operatorname{Tr}\left\{\left[H_{\nu}, H_{l}\right]^{2} \cdot\left[H_{\nu}, H_{l}^{2}\right]\right\}=2 \operatorname{Tr}\left\{\left[H_{\nu}, H_{l}\right]^{3} H_{l}\right\} \quad$ and $\operatorname{Tr}\left\{\left[H_{\nu}, H_{l}\right]^{3} H_{l}\right\}=\operatorname{Tr}\left\{\left[H_{\nu}, H_{l}\right]^{3}\right\} \cdot \operatorname{Tr}\left(H_{l}\right) / 3$. However, the WB invariant $\mathcal{I}_{1}^{(2)}$ depends on all three $C P$-violating phases in the PMNS matrix, i.e., $\{\delta, \rho, \sigma\}$; its explicit expression turns out to be quite complicated and will be omitted here.

For illustration, let us consider the possibility of radiatively generating a nontrivial value of $\delta$ via the RGE from a vanishing $\delta$ at some high-energy scale [3942]. In this case, we set $\delta=0$ as the initial condition; then the expression of $\mathcal{I}_{1}^{(2)}$ can be greatly simplified as

$$
\begin{aligned}
\mathcal{I}_{1}^{(2)}= & 2 \mathrm{i}\left\{+H_{13}\left[H_{13}\left(H_{12}^{2}-H_{23}^{2}\right)+H_{12} H_{23}\left(H_{33}-H_{11}\right)\right] m_{1} m_{3} \Delta_{12} \Delta_{23} \sin (2 \rho)\right. \\
& +H_{23}\left[H_{23}\left(H_{13}^{2}-H_{12}^{2}\right)+H_{12} H_{13}\left(H_{22}-H_{33}\right)\right] m_{3} m_{2} \Delta_{13} \Delta_{12} \sin (2 \sigma) \\
& \left.+H_{12}\left[H_{12}\left(H_{23}^{2}-H_{13}^{2}\right)+H_{23} H_{13}\left(H_{11}-H_{22}\right)\right] m_{2} m_{1} \Delta_{23} \Delta_{13} \sin (2 \rho-2 \sigma)\right\} .
\end{aligned}
$$

As $\delta$ has been set to zero, the moduli of the elements of $H_{l}$ can be directly related to three charged-lepton masses and three flavor mixing angles via

$$
\begin{aligned}
& H_{12}=s_{12} c_{12} c_{13}^{2} \Delta_{e \mu}-\left[s_{12} c_{12}\left(s_{13}^{2} c_{23}^{2}-s_{23}^{2}\right)+\left(c_{12}^{2}-s_{12}^{2}\right) s_{13} s_{23} c_{23}\right] \Delta_{\mu \tau}, \\
& H_{13}=c_{12} s_{13} c_{13} \Delta_{e \mu}-\left(s_{12} s_{23}-c_{12} s_{13} c_{23}\right) c_{13} c_{23} \Delta_{\mu \tau}, \\
& H_{23}=s_{12} s_{13} c_{13} \Delta_{e \mu}+\left(c_{12} s_{23}+s_{12} s_{13} c_{23}\right) c_{13} c_{23} \Delta_{\mu \tau}, \\
& H_{11}=m_{e}^{2}-\left(1-c_{12}^{2} c_{13}^{2}\right) \Delta_{e \mu}-\left(s_{12} s_{23}-c_{12} s_{13} c_{23}\right)^{2} \Delta_{\mu \tau}, \\
& H_{22}=m_{\mu}^{2}+s_{12}^{2} c_{13}^{2} \Delta_{e \mu}-\left(c_{12} s_{23}+s_{12} s_{13} c_{23}\right)^{2} \Delta_{\mu \tau}, \\
& H_{33}=m_{\tau}^{2}+s_{13}^{2} \Delta_{e \mu}+\left(s_{13}^{2}+c_{13}^{2} s_{23}^{2} \Delta_{\mu \tau},\right.
\end{aligned}
$$

which can be inserted back into Eq. (2.28) to obtain the explicit expression of $\mathcal{I}_{1}^{(2)}$. From Eqs. (2.26)-(2.28), we can observe that the following hold:

(i) If $C P$ is conserved (namely, $\delta=0$ and $\rho=\sigma=0$ or $\pi / 2$ ) at the initial high-energy scale, then $\mathcal{I}_{1}^{(1)}=\mathcal{I}_{1}^{(2)}=\mathcal{I}_{1}=0$ and $\mathrm{d} \mathcal{I}_{1} / \mathrm{d} t$ vanishes, implying that $\mathcal{I}_{1}$ will stay at zero all the way down to low-energy scales.

(ii) If $C P$ is violated with $\delta=0$ but nontrivial values of $\rho$ or $\sigma$ at some high-energy scale, then $\mathrm{d} \mathcal{I}_{1} / \mathrm{d} t$ is no longer vanishing, as a consequence of the nonzero $\mathcal{I}_{1}^{(2)}$ in Eq. (2.28). Consequently, as the energy scale evolves, a nonzero value of $\mathcal{I}_{1}$ will be developed, leading to a nonzero $\delta$. As stressed in Ref. [39], a nontrivial value of the Dirac $C P$ phase $\delta$ can be generated from the Majorana $C P$ phase $\rho$ or $\sigma$ via the RG running, even though $\delta=0$ is assumed at the beginning.

(b) Then, we can derive the RGE of $\mathcal{I}_{2}$ defined in Eq. (1.6) in a similar way, namely,

$$
\begin{aligned}
\frac{\mathrm{d} \mathcal{I}_{2}}{\mathrm{~d} t}= & 4\left(\alpha_{\nu}+\alpha_{l}\right) \mathcal{I}_{2} \\
& -6 \operatorname{Im}\left\{\operatorname{Tr}\left[H_{l} H_{\nu} H_{l} G_{l \nu}+H_{l} G_{l \nu}^{2}\right]\right\} / v^{2},
\end{aligned}
$$

where one can easily verify that the second term on the right-hand side actually vanishes due to the Hermiticity of $H_{l}, H_{\nu}$, and $G_{l \nu}$ and the cyclic invariance of the trace. As an immediate consequence, the derivative of the WB invariant $\mathcal{I}_{2}$ is proportional to itself. We can formally integrate Eq. (2.29) and obtain

$\mathcal{I}_{2}(t)=\mathcal{I}_{2}(0) \exp \left\{4 \int_{0}^{t}\left[\alpha_{\nu}\left(t^{\prime}\right)+\alpha_{l}\left(t^{\prime}\right)\right] \mathrm{d} t^{\prime}\right\}$,

where $\mathcal{I}_{2}(0) \equiv \mathcal{I}_{2}(t=0)$ stands for the value at the electroweak scale $\mu=\Lambda_{\mathrm{EW}}$, while $\mathcal{I}_{2}(t)$ stands for the value at an arbitrary high-energy scale $\mu=\Lambda$. For the direct connection between low- and high-energy mass or mixing parameters in an integral form, one may refer to previous works [44-47].

Since $\mathcal{I}_{2}$ depends on all the three $C P$ phases, its explicit expression is rather lengthy. As before, by setting $\delta=0$ at some energy scale, we arrive at

$$
\begin{aligned}
\mathcal{I}_{2}= & H_{13}^{2} m_{1} m_{3} \Delta_{13} \sin (2 \rho)+H_{23}^{2} m_{2} m_{3} \Delta_{23} \sin (2 \sigma) \\
& +H_{12}^{2} m_{1} m_{2} \Delta_{12} \sin (2 \rho-2 \sigma) .
\end{aligned}
$$

For $\mathcal{I}_{3}$, the RGE can be calculated easily and it is interesting to find 
$\frac{\mathrm{d} \mathcal{I}_{3}}{\mathrm{~d} t}=6\left(\alpha_{\nu}+2 \alpha_{l}\right) \mathcal{I}_{3}+9 \operatorname{Tr}\left\{\left[G_{l \nu}, H_{l}\right]^{2} \cdot\left[G_{l \nu}, H_{l}^{2}\right]\right\} / v^{2}$,

where the second term on the right-hand side is similar to $\mathcal{I}_{1}^{(1)}$ in Eq. (2.26) and the difference is simply to replace $H_{\nu}$ in the latter by $G_{l \nu}$. After a straightforward calculation, we can obtain

$$
\begin{aligned}
\operatorname{Tr}\left\{\left[G_{l \nu}, H_{l}\right]^{2} \cdot\left[G_{l \nu}, H_{l}^{2}\right]\right\} & =2 \operatorname{Tr}\left\{\left[G_{l \nu}, H_{l}\right]^{3} H_{l}\right\} \\
& =\frac{2}{3}\left(m_{e}^{2}+m_{\mu}^{2}+m_{\tau}^{2}\right) \mathcal{I}_{3},
\end{aligned}
$$

such that the RGE of $\mathcal{I}_{3}$ can be formally solved as in the case of $\mathcal{I}_{2}$, i.e.,

$$
\begin{aligned}
& \mathcal{I}_{3}(t) \\
& =\mathcal{I}_{3}(0) \exp \left\{3 \int_{0}^{t}\left[2 \alpha_{\nu}\left(t^{\prime}\right)+4 \alpha_{l}\left(t^{\prime}\right)+\sum_{\alpha} y_{\alpha}^{2}\left(t^{\prime}\right)\right] \mathrm{d} t^{\prime}\right\},
\end{aligned}
$$

where $y_{\alpha} \equiv \sqrt{2} m_{\alpha} / v$ denotes the charged-lepton Yukawa coupling for $\alpha=e, \mu, \tau$. Since the RGEs of $\alpha_{\nu}(t), \alpha_{l}(t)$, and $y_{\alpha}(t)$ can be separately solved, we establish another direct connection between the highand low-energy WB invariants.

(c) Finally, let us investigate the RGE of $\mathcal{I}_{4}$, which has been defined in Eq. (1.8). The final result is

$$
\frac{\mathrm{d} \mathcal{I}_{4}}{\mathrm{~d} t}=2\left(3 \alpha_{\nu}+2 \alpha_{l}\right) \mathcal{I}_{4}-12 \mathcal{I}_{4}^{(1)} / v^{2},
$$

where $\mathcal{I}_{4}^{(1)} \equiv \operatorname{Im}\left\{\operatorname{Tr}\left[H_{l} H_{\nu} G_{l l}^{2}\right]\right\}$ has been introduced. Notice that a few useful identities, i.e., $\operatorname{Im}\left\{\operatorname{Tr}\left[H_{l} H_{\nu}^{2} H_{l} G_{l \nu}\right]\right\}=\operatorname{Im}\left\{\operatorname{Tr}\left[H_{l} G_{l \nu} H_{\nu} G_{l \nu}\right]\right\}=0$ and $\operatorname{Tr}\left[H_{l} H_{\nu} H_{l} H_{\nu} G_{l \nu}\right]=\operatorname{Tr}\left[H_{l} H_{\nu} G_{l \nu}^{2}\right]$, have been used. Because of the second term on the right-hand side of Eq. (2.35), it is not possible to directly solve the RGE of $\mathcal{I}_{4}$. To render the analytical formulas of $\mathcal{I}_{4}$ and $\mathcal{I}_{4}^{(1)}$ readable, we set $\delta=0$ and get

$$
\begin{aligned}
\mathcal{I}_{4}= & +H_{13}^{2} m_{1} m_{3} \Delta_{13}\left(m_{1}^{2}+m_{3}^{2}\right) \sin (2 \rho) \\
& +H_{23}^{2} m_{2} m_{3} \Delta_{23}\left(m_{2}^{2}+m_{3}^{2}\right) \sin (2 \sigma) \\
& +H_{12}^{2} m_{1} m_{2} \Delta_{12}\left(m_{1}^{2}+m_{2}^{2}\right) \sin (2 \rho-2 \sigma)
\end{aligned}
$$

and

$$
\begin{aligned}
\mathcal{I}_{4}^{(1)}= & +H_{13}\left[H_{12} H_{23}+H_{13}\left(H_{11}+H_{33}\right)\right] m_{1} m_{3} \Delta_{13}\left(m_{1}^{2}+m_{3}^{2}\right) \sin (2 \rho) \\
& +H_{23}\left[H_{12} H_{13}+H_{23}\left(H_{22}+H_{33}\right)\right] m_{2} m_{3} \Delta_{23}\left(m_{2}^{2}+m_{3}^{2}\right) \sin (2 \sigma) \\
& +H_{12}\left[H_{13} H_{23}+H_{12}\left(H_{11}+H_{22}\right)\right] m_{1} m_{2} \Delta_{12}\left(m_{1}^{2}+m_{2}^{2}\right) \sin (2 \rho-2 \sigma) .
\end{aligned}
$$

Given $\mathcal{I}_{1}=0$ or equivalently $\delta=0$, we can see that $\mathcal{I}_{2}$ in Eq. (2.31), $\mathcal{I}_{4}$, and $\mathcal{I}_{4}^{(1)}$ are vanishing if $\rho$ and $\sigma$ take trivial values of 0 or $\pi / 2$ at the beginning. This is also true for $\mathcal{I}_{3}$, although its expression has not been explicitly written down.

To conclude, we find that $\mathrm{d} \mathcal{I}_{2} / \mathrm{d} t=4\left(\alpha_{\nu}+\alpha_{l}\right) \mathcal{I}_{2}$ and $\mathrm{d} \mathcal{I}_{3} / \mathrm{d} t=3\left[2 \alpha_{\nu}+4 \alpha_{l}+\left(y_{e}^{2}+y_{\mu}^{2}+y_{\tau}^{2}\right)\right] \mathcal{I}_{3}$, which can be formally solved, and thus establish a direct link between low- and high-energy WB invariants. For $\mathcal{I}_{1}$ and $\mathcal{I}_{4}$, their derivatives with respect to $t=\left[\ln \left(\mu / \Lambda_{\mathrm{EW}}\right)\right] /\left(16 \pi^{2}\right)$ turn out not to be proportional to themselves. However, if $C P$ conservation is assumed at some energy scale, i.e., all three $C P$ phases take trivial values, then $C P$ will be conserved all the way down to the electroweak scale. If one of the three $C P$ phases is nontrivial at the beginning, namely, $C P$ violation exists in theory, the other phases will be generated radiatively during the RGE running. In the case of partial or complete neutrino mass degeneracy, one can choose suitable WB invariants from $\left\{\mathcal{I}_{1}, \mathcal{I}_{2}, \mathcal{I}_{3}, \mathcal{I}_{4}\right\}$ and apply the corresponding RGEs to study their running behaviors.

\section{CANONICAL SEESAW MODEL}

The partial or complete mass degeneracy of three light neutrinos has already been excluded by neutrino oscillation data $[48,49]$, which require two independent neutrino mass-squared differences to be $\Delta_{21} \approx 7.4 \times 10^{-5} \mathrm{eV}^{2}$ and $\Delta_{31} \approx \pm 2.5 \times 10^{-3} \mathrm{eV}^{2}$. On the other hand, as we have mentioned, the effective theory considered in the previous section is valid when the heavy degrees of freedom associated with neutrino mass generation are integrated out. Therefore, we now examine the necessary and sufficient conditions for $C P$ conservation with a partial or complete mass degeneracy of three heavy Majorana neutrinos in the canonical seesaw model, for which the gauge-invariant Lagrangian was given in Eq. (1.1). After the spontaneous gauge symmetry breaking, it can be rewritten as

$$
\begin{aligned}
\mathcal{L}_{\text {lepton }}= & -\overline{l_{\mathrm{L}}} M_{l} l_{\mathrm{R}}-\overline{\nu_{\mathrm{L}}} M_{\mathrm{D}} N_{\mathrm{R}}-\frac{1}{2} \overline{N_{\mathrm{R}}^{\mathrm{C}}} M_{\mathrm{R}} N_{\mathrm{R}} \\
& +\frac{g}{\sqrt{2}} \overline{l_{\mathrm{L}}} \gamma^{\mu} \nu_{\mathrm{L}} W_{\mu}^{-}+\text {H.c. },
\end{aligned}
$$


where the charged-current interaction has been included to cover all possible places for $C P$ violation. In the presence of right-handed neutrinos, the sufficient and necessary conditions for $C P$ conservation in the full seesaw model are equivalent to the existence of three unitary matrices $U_{\mathrm{L}}, U_{\mathrm{R}}$, and $V_{\mathrm{R}}$ such that the Lagrangian in Eq. (3.1) is invariant under

$$
\begin{aligned}
& l_{\mathrm{L}} \rightarrow U_{\mathrm{L}} \mathcal{C} l_{\mathrm{L}}^{*}, \quad \nu_{\mathrm{L}} \rightarrow U_{\mathrm{L}} \mathcal{C} \nu_{\mathrm{L}}^{*}, \quad l_{\mathrm{R}} \rightarrow U_{\mathrm{R}} \mathcal{C} l_{\mathrm{R}}^{*}, \\
& N_{\mathrm{R}} \rightarrow V_{\mathrm{R}} \mathcal{C} N_{\mathrm{R}}^{*}, \quad W_{\mu}^{-} \rightarrow-(-1)^{\delta_{0 \mu}} W_{\mu}^{+} \text {, }
\end{aligned}
$$

where the notation is the same as in Eq. (1.3). In terms of the fermion mass matrices, one can easily prove that this is equivalent to the conditions

$$
\begin{aligned}
U_{\mathrm{L}}^{\dagger} M_{l} U_{\mathrm{R}} & =M_{l}^{*}, \quad U_{\mathrm{L}}^{\dagger} M_{\mathrm{D}} V_{\mathrm{R}}=M_{\mathrm{D}}^{*}, \\
V_{\mathrm{R}}^{\mathrm{T}} M_{\mathrm{R}} V_{\mathrm{R}} & =-M_{\mathrm{R}}^{*},
\end{aligned}
$$

which will be used to construct the WB invariants for $C P$ conservation, similar to the construction in the effective theory. To this end, we further introduce $H_{\mathrm{D}} \equiv M_{\mathrm{D}}^{\dagger} M_{\mathrm{D}}$, $H_{\mathrm{R}} \equiv M_{\mathrm{R}}^{\dagger} M_{\mathrm{R}}, G_{\mathrm{DR}} \equiv M_{\mathrm{R}}^{\dagger} H_{\mathrm{D}}^{*} M_{\mathrm{R}}$, and

$$
H_{n} \equiv M_{\mathrm{D}}^{\dagger}\left(H_{l}\right)^{n} M_{\mathrm{D}}, \quad G_{n} \equiv M_{\mathrm{R}}^{\dagger} H_{n}^{*} M_{\mathrm{R}},
$$

where $n$ denotes the positive integer. It is straightforward to verify that the transformation rules for these newly defined Hermitian matrices are as follows:

$$
\begin{array}{lll}
V_{\mathrm{R}}^{\dagger} H_{\mathrm{D}} V_{\mathrm{R}}=H_{\mathrm{D}}^{*}, & V_{\mathrm{R}}^{\dagger} H_{\mathrm{R}} V_{\mathrm{R}}=H_{\mathrm{R}}^{*}, & V_{\mathrm{R}}^{\dagger} G_{\mathrm{DR}} V_{\mathrm{R}}=G_{\mathrm{DR}}^{*}, \\
V_{\mathrm{R}}^{\dagger} H_{n} V_{\mathrm{R}}=H_{n}^{*}, & V_{\mathrm{R}}^{\dagger} G_{n} V_{\mathrm{R}}=G_{n}^{*},
\end{array}
$$

which are universal and make the construction of WB invariants much easier. As shown in Ref. [20], the sufficient and necessary conditions for $C P$ conservation are equivalent to the vanishing of a minimal set of WB invariants.

Before constructing the WB invariants, we count the number of physical parameters in the canonical seesaw model and will pay particular attention to the $C P$ phases. Without loss of generality, one can always choose the basis in which $M_{l}, M_{\mathrm{R}}$ and the charged-current interaction in Eq. (3.1) are simultaneously diagonal, so that the complex mass matrix $M_{\mathrm{D}}$ will be the only source of $C P$ violation. Following Refs. [20,50], we adopt the convenient parametrization $M_{\mathrm{D}}=U_{\mathrm{D}} Y_{\Delta}$, where $U_{\mathrm{D}}$ is a $3 \times 3$ unitary matrix and $Y_{\Delta}$ is a lower triangular matrix, i.e.,

$$
Y_{\Delta}=\left(\begin{array}{ccc}
y_{11} & 0 & 0 \\
y_{21} e^{\mathrm{i} \phi_{21}} & y_{22} & 0 \\
y_{31} e^{\mathrm{i} \phi_{31}} & y_{32} e^{\mathrm{i} \phi_{32}} & y_{33}
\end{array}\right),
$$

where $y_{i j}$ (for $1 \leq j \leq i \leq 3$ ) are all real and positive parameters and $\phi_{i j}$ (for $i j=21,31,32$ ) are the phases of three off-diagonal nonzero elements. As usual, three unphysical phases of $U_{\mathrm{D}}$ can be eliminated by redefining the phases of $\nu_{\mathrm{L}}, l_{\mathrm{L}}$, and $l_{\mathrm{R}}$, leaving the Lagrangian unchanged. Therefore, $M_{\mathrm{D}}$ contains only 15 real parameters, six of which are phases. To be more explicit, we rewrite it as $M_{\mathrm{D}}=U_{\xi} P_{\alpha} Y_{\zeta} P_{\beta}$ [20], where $P_{\alpha}=$ $\operatorname{diag}\left\{1, e^{\mathrm{i} \alpha_{1}}, e^{\mathrm{i} \alpha_{2}}\right\}$ and $P_{\beta}=\operatorname{diag}\left\{1, e^{\mathrm{i} \beta_{1}}, e^{\mathrm{i} \beta_{2}}\right\}$ are two diagonal phase matrices. In addition,

$$
Y_{\zeta}=\left(\begin{array}{ccc}
y_{11} & 0 & 0 \\
y_{21} & y_{22} & 0 \\
y_{31} & y_{32} e^{\mathrm{i} \zeta} & y_{33}
\end{array}\right)
$$

is related to $Y_{\Delta}$ by properly factorizing out relevant phases, and $U_{\xi}$ is the Cabibbo-Kobayashi-Maskawa (CKM)-like unitary matrix, with $\xi$ being the $C P$ phase and the three rotation angles being $\left\{\theta_{12}^{\mathrm{D}}, \theta_{13}^{\mathrm{D}}, \theta_{23}^{\mathrm{D}}\right\}$. In this way, 15 real parameters of $M_{\mathrm{D}}$ are now specified, i.e., six phases $\left\{\xi, \zeta, \alpha_{1}, \alpha_{2}, \beta_{1}, \beta_{2}\right\}$ and nine real parameters $\left\{\theta_{12}^{\mathrm{D}}, \theta_{13}^{\mathrm{D}}, \theta_{23}^{\mathrm{D}}\right\}$ and $\left\{y_{11}, y_{22}, y_{33}, y_{21}, y_{31}, y_{32}\right\}$. All the information about $C P$ violation is represented by six phases of $M_{\mathrm{D}}$. As we shall show soon, $C P$ is conserved if and only if $\sin \alpha_{1}=\sin \alpha_{2}=\sin \xi=\sin \zeta=\sin 2 \beta_{1}=\sin 2 \beta_{2}=0$ holds. ${ }^{5}$ Using the adopted parametrization of $M_{\mathrm{D}}$, we can obtain

$$
H_{\mathrm{D}}=M_{\mathrm{D}}^{\dagger} M_{\mathrm{D}}=P_{\beta}^{\dagger} Y_{\zeta}^{\dagger} Y_{\zeta} P_{\beta},
$$

where only three phases $\left\{\zeta, \beta_{1}, \beta_{2}\right\}$ are involved. Consequently, even if $H_{\mathrm{D}}$ were real, there would still be $C P$ violation. Unlike the effective theory, in which real $H_{l}=M_{l} M_{l}^{\dagger}$ implies $C P$ conservation, all six phases of $M_{\mathrm{D}}$ are important [20].

\section{A. Nondegenerate masses}

First, we summarize the main results in the case of nondegenerate masses, namely, $M_{1} \neq M_{2} \neq M_{3}$, where $M_{i}$ stands for the heavy Majorana neutrino mass (for $i=1,2,3)$. As demonstrated in Ref. [20], the following six conditions,

$$
\begin{gathered}
\tilde{\mathcal{I}}_{1} \equiv \operatorname{Im}\left\{\operatorname{Tr}\left[H_{\mathrm{D}} H_{\mathrm{R}} G_{\mathrm{DR}}\right]\right\}=0, \\
\tilde{\mathcal{I}}_{2} \equiv \operatorname{Im}\left\{\operatorname{Tr}\left[H_{\mathrm{D}} H_{\mathrm{R}}^{2} G_{\mathrm{DR}}\right]\right\}=0, \\
\tilde{\mathcal{I}}_{3} \equiv \operatorname{Im}\left\{\operatorname{Tr}\left[H_{\mathrm{D}} H_{\mathrm{R}}^{2} G_{\mathrm{DR}} H_{\mathrm{R}}\right]\right\}=0, \\
\tilde{\mathcal{I}}_{4} \equiv \operatorname{Im}\left\{\operatorname{Tr}\left[H_{1} H_{\mathrm{R}} G_{1}\right]\right\}=0, \\
\tilde{\mathcal{I}}_{5} \equiv \operatorname{Im}\left\{\operatorname{Tr}\left[H_{1} H_{\mathrm{R}}^{2} G_{1}\right]\right\}=0, \\
\tilde{\mathcal{I}}_{6} \equiv \operatorname{Im}\left\{\operatorname{Tr}\left[H_{1} H_{\mathrm{R}}^{2} G_{1} H_{\mathrm{R}}\right]\right\}=0,
\end{gathered}
$$

\footnotetext{
${ }^{5}$ It is worth noticing that $\beta_{1}$ and $\beta_{2}$ are actually the Majoranatype $C P$ phases and can take the value of $\pi / 2$ without violating the $C P$ symmetry.
} 
must be fulfilled to guarantee $C P$ conservation. Notice that $H_{n}$ and $G_{n}$ [having been introduced in Eq. (3.4)] with $n=1$ have been used in Eqs. (3.12)-(3.14). With the help of the transformation rules in Eq. (3.5), we can easily prove that $\tilde{\mathcal{I}}_{i}(i=1,2, \ldots, 6)$ are WB invariants and $\tilde{\mathcal{I}}_{i}=0$ in Eqs. (3.9)-(3.14) serve as the sufficient and necessary conditions for $C P$ conservation.

Since $\left\{\tilde{\mathcal{I}}_{1}, \tilde{\mathcal{I}}_{2}, \tilde{\mathcal{I}}_{3}\right\}$ depend on only three phases in $H_{\mathrm{D}}$, i.e., $\left\{\zeta, \beta_{1}, \beta_{2}\right\}$, the vanishing of these three WB invariants gives three independent constraints on the relevant three phases. The other WB invariants $\left\{\tilde{\mathcal{I}}_{4}, \tilde{\mathcal{I}}_{5}, \tilde{\mathcal{I}}_{6}\right\}$ depend on all six phases in $M_{\mathrm{D}}$. After three phases in $H_{\mathrm{D}}$ are eliminated by Eqs. (3.9)-(3.11), we are left with another set of three independent constraints from Eqs. (3.12)-(3.14) on the remaining three phases, i.e., $\left\{\xi, \alpha_{1}, \alpha_{2}\right\}$. However, as explained in Ref. [23], although $\left\{\tilde{\mathcal{I}}_{1}, \tilde{\mathcal{I}}_{2}, \tilde{\mathcal{I}}_{3}\right\}$ are independent, the vanishing of them leads to three nonlinear equations of $\zeta, \beta_{1}$, and $\beta_{2}$, from which nontrivial solutions (i.e., those other than 0 and $\pi / 2$ ) of these three phases can be obtained for some special values of other physical parameters. For this reason, we recommend another set of three invariants $\left\{\tilde{\mathcal{I}}_{1}, \tilde{\mathcal{I}}_{2}, \tilde{\mathcal{I}}_{3}^{\prime}\right\}$, where the new WB invariant $\tilde{\mathcal{I}}_{3}^{\prime} \equiv \operatorname{Tr}\left\{\left[H_{\mathrm{R}}, H_{\mathrm{D}}\right]^{3}\right\}$ replaces the original one $\tilde{\mathcal{I}}_{3}$. In the chosen basis, where $H_{\mathrm{R}}=\operatorname{diag}\left\{M_{1}^{2}, M_{2}^{2}, M_{3}^{2}\right\}$ and $H_{\mathrm{D}}$ is as given in Eq. (3.8), one can explicitly find

$$
\tilde{\mathcal{I}}_{3}^{\prime}=6\left(M_{1}^{2}-M_{2}^{2}\right)\left(M_{1}^{2}-M_{3}^{2}\right)\left(M_{2}^{2}-M_{3}^{2}\right) y_{22} y_{33}^{2} y_{21} y_{31} y_{32} \sin \zeta,
$$

which is simply proportional to $\sin \zeta$. If the masses of heavy Majorana neutrinos are nondegenerate and the parameters $y_{i j}$ are nonzero, then $\tilde{\mathcal{I}}_{3}^{\prime}=0$ is the sufficient and necessary condition for $\zeta=0$. Now that $\zeta=0$ is guaranteed by $\tilde{\mathcal{I}}_{3}^{\prime}=0$, we can calculate the other two invariants, namely,

$$
\begin{aligned}
\tilde{\mathcal{I}}_{1}= & M_{2}^{3}\left[M_{3} y_{33}^{2} y_{32}^{2}+M_{1}\left(y_{22} y_{21}+y_{31} y_{32}\right)^{2}+M_{2}\left(y_{22}^{2}+y_{32}^{2}\right)^{2}\right] \sin 2 \beta_{1} \\
& +M_{3}^{3} y_{33}^{2}\left(M_{3} y_{33}^{2}+M_{1} y_{31}^{2}+M_{2} y_{32}^{2}\right) \sin 2 \beta_{2}=0, \\
\tilde{\mathcal{I}}_{2}= & M_{2}^{5}\left[M_{3} y_{33}^{2} y_{32}^{2}+M_{1}\left(y_{22} y_{21}+y_{31} y_{32}\right)^{2}+M_{2}\left(y_{22}^{2}+y_{32}^{2}\right)^{2}\right] \sin 2 \beta_{1} \\
& +M_{3}^{5} y_{33}^{2}\left(M_{3} y_{33}^{2}+M_{1} y_{31}^{2}+M_{2} y_{32}^{2}\right) \sin 2 \beta_{2}=0 .
\end{aligned}
$$

The above system of linear homogeneous equations of $\sin 2 \beta_{1}$ and $\sin 2 \beta_{2}$ has the unique trivial solutions $\sin 2 \beta_{1}=0$ and $\sin 2 \beta_{2}=0$, since the determinant of the coefficient matrix is proportional to $\left(M_{3}^{2}-M_{2}^{2}\right)$, which is nonzero in the case of nondegenerate masses. Therefore, the vanishing of three WB invariants $\left\{\tilde{\mathcal{I}}_{1}, \tilde{\mathcal{I}}_{2}, \tilde{\mathcal{I}}_{3}^{\prime}\right\}$ is the sufficient and necessary condition for the vanishing of those three phases in $H_{\mathrm{D}}$. After fixing three phases in $H_{\mathrm{D}}$, we have another three independent constraints on the remaining phases $\left\{\xi, \alpha_{1}, \alpha_{2}\right\}$ from Eqs. (3.12)-(3.14). However, these equations are in general nonlinear, so there may be some parameter space where Eqs. (3.12)-(3.14) do not necessarily imply $C P$ conservation, as shown in Ref. [23] for the effective theory. Without any information about the physical parameters at high-energy scales, such as the heavy Majorana neutrino masses and the matrix elements of $M_{\mathrm{D}}$, it is impossible for us to find another set of three invariants to guarantee $C P$ conservation, at least in the physically allowed parameter. Therefore, we take Eqs. (3.12)-(3.14) to be the sufficient and necessary conditions of eliminating the remaining three phases in a particular parameter space.

Although the invariants given in Eqs. (3.9)-(3.14) are by construction independent of the flavor basis, it is convenient to calculate them in the special basis where $M_{l}$ and $M_{\mathrm{R}}$ are both diagonal. By inspecting these conditions, we can prove that $C P$ symmetry is conserved if and only if

$$
\begin{aligned}
\omega_{m n}^{\alpha} & \equiv \arg \left[\left(M_{\mathrm{D}}\right)_{\alpha m}\right]-\arg \left[\left(M_{\mathrm{D}}\right)_{\alpha n}\right] \\
& =\left(p_{n}-p_{m}\right) \frac{\pi}{2}+k_{\alpha} \pi,
\end{aligned}
$$

where $p_{n}, p_{m}, k_{\alpha}$ are arbitrary integers with $m, n=1,2,3$ and $\alpha=e, \mu, \tau$. The above equation gives in total six independent constraints on the phases of $M_{\mathrm{D}}$, while the number of independent phases in $M_{\mathrm{D}}$ responsible for $C P$ violation is also six. From Eq. (3.18), we conclude that in the basis where $M_{l}$ and $M_{\mathrm{R}}$ are diagonal, if the masses of heavy Majorana neutrinos are nondegenerate, then the sufficient and necessary conditions for $C P$ conservation are simply that (i) the phases of the elements of $M_{\mathrm{D}}$ in the same row but different columns can differ only by an integral multiple of $\pi / 2$, and (ii) the phase differences between two different rows, i.e., $\omega_{m n}^{\alpha}-\omega_{m n}^{\beta}$, can differ only by an even multiple of $\pi / 2$.

As a concrete example for the $C P$ violation at highenergy scales, we consider the $C P$-violating decays of heavy Majorana neutrinos into left-handed lepton and Higgs doublets, i.e., $N_{i} \rightarrow \ell_{\alpha}+H$ and $N_{i} \rightarrow \bar{\ell}_{\alpha}+\bar{H}$ (for $i=1,2,3$ and $\alpha=e, \mu, \tau)$. The $C P$ asymmetries arise from the interference between the tree-level and one-loop-level decay amplitudes and can be written as 
$\epsilon_{i \alpha} \equiv \frac{\Gamma\left(N_{i} \rightarrow \ell_{\alpha}+H\right)-\Gamma\left(N_{i} \rightarrow \bar{\ell}_{\alpha}+\bar{H}\right)}{\sum_{\alpha}\left[\Gamma\left(N_{i} \rightarrow \ell_{\alpha}+H\right)+\Gamma\left(N_{i} \rightarrow \bar{\ell}_{\alpha}+\bar{H}\right)\right]}$,

where $\Gamma\left(N_{i} \rightarrow \ell_{\alpha}+H\right)$ and $\Gamma\left(N_{i} \rightarrow \bar{\ell}_{\alpha}+\bar{H}\right)$ stand for the decay rate of $N_{i} \rightarrow \ell_{\alpha}+H$ and that of $N_{i} \rightarrow \bar{\ell}_{\alpha}+\bar{H}$, respectively. In the vanilla scenario of leptogenesis, the $C P$ violation in the out-of-equilibrium decays of heavy Majorana neutrinos gives rise to lepton number asymmetries, which will be finally converted into baryon number asymmetry in our Universe [14-16]. Concentrating on the $C P$ asymmetries, in the basis where $M_{l}$ and $M_{\mathrm{R}}$ are diagonal, we have [41]

$$
\begin{aligned}
\epsilon_{i \alpha}= & \frac{1}{4 \pi v^{2}\left(H_{\mathrm{D}}\right)_{i i}} \sum_{j \neq i}\left\{\operatorname{Im}\left[\left(M_{\mathrm{D}}^{*}\right)_{\alpha i}\left(M_{\mathrm{D}}\right)_{\alpha j}\left(H_{\mathrm{D}}\right)_{i j}\right] \mathcal{F}\left(\frac{M_{j}^{2}}{M_{i}^{2}}\right)\right. \\
& \left.+\operatorname{Im}\left[\left(M_{\mathrm{D}}^{*}\right)_{\alpha i}\left(M_{\mathrm{D}}\right)_{\alpha j}\left(H_{\mathrm{D}}\right)_{i j}^{*}\right] \mathcal{G}\left(\frac{M_{j}^{2}}{M_{i}^{2}}\right)\right\},
\end{aligned}
$$

where the loop functions $\mathcal{F}(x) \equiv \sqrt{x}\{(2-x) /(1-x)+$ $(1+x) \ln [x /(1+x)]\}$ and $\mathcal{G}(x) \equiv 1 /(1-x)$ have been defined. It is easy to verify that all the $C P$ asymmetries $\epsilon_{i \alpha}$ (for $i=1,2,3$ and $\alpha=e, \mu, \tau$ ) vanish if the phases of the matrix elements of $M_{\mathrm{D}}$ satisfy the following relations:

$$
\sin \left(\omega_{i j}^{\alpha}+\omega_{i j}^{\beta}\right)=\sin \left(\omega_{i j}^{\alpha}-\omega_{i j}^{\beta}\right)=0,
$$

where $\omega_{i j}^{\alpha} \equiv \arg \left[\left(M_{\mathrm{D}}\right)_{\alpha i}\right]-\arg \left[\left(M_{\mathrm{D}}\right)_{\alpha j}\right]$ has been defined, and likewise for $\omega_{i j}^{\beta}$. The solutions to Eq. (3.21) are exactly the same as those in Eq. (3.18). Hence we reach the conclusion that if the phases of the matrix elements of $M_{\mathrm{D}}$ fulfill the conditions in Eq. (3.18) in the basis where $M_{l}$ and $M_{\mathrm{R}}$ are diagonal, then there will be no $C P$ violation in the canonical seesaw model and all the $C P$ asymmetries $\epsilon_{i \alpha}$ vanish in the decays of heavy Majorana neutrinos.

In summary, if the masses of heavy Majorana neutrinos are nondegenerate, we must implement six WB invariants to ensure $C P$ conservation, e.g., those in Eqs. (3.9)-(3.14). This conclusion has been obtained in the literature $[20,26]$. However, if a partial or complete mass degeneracy of heavy Majorana neutrinos is assumed, an immediate question is how many WB invariants we need for $C P$ conservation.

\section{B. Partial mass degeneracy}

If the masses of heavy Majorana neutrinos are partially degenerate, e.g., $M_{1}=M_{2} \neq M_{3}$, then one can verify that $\left\{\tilde{\mathcal{I}}_{1}, \tilde{\mathcal{I}}_{2}, \tilde{\mathcal{I}}_{3}\right\}$ become linearly dependent on each other, and so do $\left\{\tilde{\mathcal{I}}_{4}, \tilde{\mathcal{I}}_{5}, \tilde{\mathcal{I}}_{6}\right\}$. As a consequence, Eqs. (3.9)-(3.14) give rise to only two independent equations, which are insufficient to guarantee $C P$ conservation. In this section, we attempt to make clear how many $C P$ phases are left in the theory and how to construct the WB invariants for $C P$ conservation in the presence of a partial mass degeneracy.
First, in the basis where $M_{l}$ and $M_{\mathrm{R}}$ are diagonal, we have the freedom to rotate the heavy Majorana neutrino fields as $N_{\mathrm{R}} \rightarrow R_{12}^{\dagger}(\alpha) N_{\mathrm{R}}$, where $R_{12}(\alpha)$ is the same rotation matrix as that given in Sec. II A. Under such a rotation, we have

$M_{\mathrm{D}} \rightarrow M_{\mathrm{D}} R_{12}(\alpha), \quad H_{\mathrm{D}} \rightarrow R_{12}^{\dagger}(\alpha) H_{\mathrm{D}} R_{12}(\alpha)$,

while the charged-lepton mass matrix $M_{l}$ and the chargedcurrent interaction are unchanged. Similar to what we have done in Sec. II A, one can adjust $\alpha$ to eliminate one of three $C P$ phases in $H_{\mathrm{D}}$, e.g., $\beta_{2}$. Hence with only two phases left in $H_{\mathrm{D}}$, the expression of $\tilde{\mathcal{I}}_{1}$ becomes quite simple

$$
\tilde{\mathcal{I}}_{1}=M_{1} M_{3}\left(M_{1}^{2}-M_{3}^{2}\right) y_{33}^{2} y_{32}^{2} \sin \left[2\left(\beta_{1}+\zeta\right)\right] .
$$

In addition to $\tilde{\mathcal{I}}_{1}$, inspired by $\mathcal{I}_{3}$ in Eq. (1.7), we introduce another WB invariant that depends only on the phases in $H_{\mathrm{D}}$, namely,

$$
\tilde{\mathcal{I}}_{7} \equiv \operatorname{Tr}\left\{\left[G_{\mathrm{DR}}, H_{\mathrm{D}}\right]^{3}\right\}=0 .
$$

After some algebraic calculations, it is easy to verify that $\tilde{\mathcal{I}}_{1}=0$ leads to either $\beta_{1}+\zeta=0$ or $\beta_{1}+\zeta=\pi / 2$, as indicated by Eq. (3.23). Furthermore, we can obtain $\tilde{\mathcal{I}}_{7} \propto$ $\sin \beta_{1}$ in the former case, while $\tilde{\mathcal{I}}_{7} \propto \cos \beta_{1}$ in the latter. Therefore, $\tilde{\mathcal{I}}_{1}=0$ and $\tilde{\mathcal{I}}_{7}=0$ imply either $\beta_{1}=\beta_{2}=\sigma=0$ or $\beta_{1}=\pi / 2, \beta_{2}=\zeta=0$, rendering three $C P$ phases $H_{\mathrm{D}}$ trivial.

Then, we need another three independent invariants to eliminate the remaining three phases in $M_{\mathrm{D}}$. However, as mentioned above, $\left\{\tilde{\mathcal{I}}_{4}, \tilde{\mathcal{I}}_{5}, \tilde{\mathcal{I}}_{6}\right\}$ turn out to be linearly dependent in the case of a partial mass degeneracy, so they are no longer sufficient to give three independent constraints on the $C P$ phases in $M_{\mathrm{D}}$. To this end, we shall construct a new series of WB invariants by using $H_{n}$ and $G_{n}$, which were introduced in Eq. (3.4). For instance, we introduce

$$
\begin{aligned}
& \tilde{\mathcal{I}}_{8} \equiv \operatorname{Tr}\left\{\left[G_{1}, H_{1}\right]^{3}\right\}=0, \\
& \tilde{\mathcal{I}}_{9} \equiv \operatorname{Tr}\left\{\left[G_{2}, H_{2}\right]^{3}\right\}=0, \\
& \tilde{\mathcal{I}}_{10} \equiv \operatorname{Tr}\left\{\left[G_{3}, H_{3}\right]^{3}\right\}=0,
\end{aligned}
$$

where the explicit expressions of $H_{n}$ and $G_{n}$ (for $n=1,2,3$ ) can be read off from Eq. (3.4). The construction of three WB invariants in Eqs. (3.25)-(3.27) has been inspired by two important observations. First, all the invariants $\left\{\tilde{\mathcal{I}}_{8}, \tilde{\mathcal{I}}_{9}, \tilde{\mathcal{I}}_{10}\right\}$ are constructed by directly using $M_{\mathrm{D}}$ instead of $H_{\mathrm{D}}$, so these invariants contain the remaining three $C P$ phases in $M_{\mathrm{D}}$. Second, these invariants are similar to each other but have been constructed intentionally by adopting the charged-lepton mass matrix via $\left(H_{l}\right)^{n}$ for $n=1,2,3$. 
In this way, because of the hierarchical mass spectrum of charged leptons, these three invariants are linearly independent even when the masses of heavy Majorana neutrinos are fully degenerate. Therefore, one can constrain the remaining phases in $M_{\mathrm{D}}$ to be trivial by requiring $\tilde{\mathcal{I}}_{8}=\tilde{\mathcal{I}}_{9}=\tilde{\mathcal{I}}_{10}=0$, whereas those three $C P$ phases in $H_{\mathrm{D}}$ have already been eliminated by $\tilde{\mathcal{I}}_{1}=\tilde{\mathcal{I}}_{7}=0$.

To conclude, in the presence of a partial mass degeneracy of heavy Majorana neutrinos, the number of $C P$ phases in the theory will be reduced from six to five. In this case, we advocate a new set of WB invariants $\left\{\tilde{\mathcal{I}}_{1}, \tilde{\mathcal{I}}_{7}, \tilde{\mathcal{I}}_{8}, \tilde{\mathcal{I}}_{9}, \tilde{\mathcal{I}}_{10}\right\}$. The vanishing of all these invariants serves as the sufficient and necessary condition for $C P$ conservation in this particular case.

\section{Complete mass degeneracy}

Once the masses of heavy Majorana neutrinos are completely degenerate, i.e., $M_{1}=M_{2}=M_{3}$, all six WB invariants in Eqs. (3.9)-(3.14) will automatically vanish. Therefore, they will not carry any useful information about $C P$ violation.

In the presence of full mass degeneracy, however, we are allowed to perform an arbitrary orthogonal rotation of $M_{\mathrm{R}}$ in the basis where both $M_{l}$ and $M_{\mathrm{R}}$ are diagonal without changing the heavy Majorana neutrino mass term. As we have proved in Sec. II B, these 3 degrees of freedom in the arbitrary orthogonal rotation can be taken to reduce the number of $C P$ phases in $H_{\mathrm{D}}$ at most by two, so we are left with four $C P$ phases in total.

It is obvious that these four $C P$ phases can be made trivial by requiring four WB invariants in Eq. (3.24) and in Eqs. (3.25)-(3.27) to be zero. First, $\tilde{\mathcal{I}}_{7}=0$ can be used to get rid of the only $C P$ phase in $H_{\mathrm{D}}$, as the other two phases have been removed by two successive rotations. Then, the vanishing of $\left\{\tilde{\mathcal{I}}_{8}, \tilde{\mathcal{I}}_{9}, \tilde{\mathcal{I}}_{10}\right\}$ in Eqs. (3.25)-(3.27) guarantees that the three remaining phases in $M_{\mathrm{D}}$ are trivial. Therefore, for the complete mass degeneracy of heavy Majorana neutrinos, there are four $C P$ phases and the vanishing of the WB invariants $\left\{\tilde{\mathcal{I}}_{7}, \tilde{\mathcal{I}}_{8}, \tilde{\mathcal{I}}_{9}, \tilde{\mathcal{I}}_{10}\right\}$ serves as the sufficient and necessary condition for $C P$ conservation.

It is worth stressing that the partial or complete mass degeneracy of heavy Majorana neutrinos may be guaranteed by flavor symmetries or may simply be accidental, and thus the degeneracy will be shifted by explicit symmetry breaking or radiative corrections [51-56], leading to the possibility of successful resonant leptogenesis [57,58]. Moreover, once the mass degeneracy is broken, the number of $C P$-violating phases and relevant $\mathrm{WB}$ invariants will be changed, as discussed in Sec. III A.

In the presence of either partial or complete mass degeneracy of heavy Majorana neutrinos, the $C P$ asymmetries defined in Eq. (3.19) cannot simply be obtained from Eq. (3.20), which turns out to be singular in the exact degeneracy limit (i.e., $M_{i}=M_{j}$ ). When the resonant mixing between any two nearly degenerate unstable particles is properly treated [19,57-60], the divergence arising from one-loop self-energy corrections to the heavy Majorana neutrino decays can be removed. After taking into account both one-loop self-energy and vertex corrections, one can find that the loop functions in the expressions of $C P$ asymmetries in Eq. (3.20) are modified with a regulator [61],

$$
\begin{aligned}
\mathcal{F}\left(x_{i j}\right) & =\sqrt{x_{i j}}\left[\frac{1-x_{i j}}{\left(1-x_{i j}\right)^{2}+r_{i j}^{2}}+1+\left(1+x_{i j}\right) \ln \left(\frac{x_{i j}}{1+x_{i j}}\right)\right], \\
\mathcal{G}\left(x_{i j}\right) & =\frac{1-x_{i j}}{\left(1-x_{i j}\right)^{2}+r_{i j}^{2}},
\end{aligned}
$$

where $x_{i j} \equiv M_{i}^{2} / M_{j}^{2}$ has been defined and the regulator $r_{i j}$ has been introduced [57-60]. If the mass spectrum of heavy Majorana neutrinos is strongly hierarchical, then the loop functions defined in Eq. (3.28) will be reduced to the forms below Eq. (3.20). However, when the masses of heavy Majorana neutrinos become nearly degenerate, the regulator $r_{i j}$ will play an important role. In particular, in the limit of exact mass degeneracy, i.e., $x_{i j}=1$, the regulator removes the singularity and gives a physically meaningful result.

It should be emphasized that in the limit of complete mass degeneracy (i.e., $M_{1}=M_{2}=M_{3}$ ), although the $C P$ asymmetries $\epsilon_{i \alpha}$ defined in Eq. (3.19) remain nonvanishing due to the contribution from the interference between the tree-level amplitude and the one-loop vertex correction, there are actually no $C P$ asymmetries in the decays of heavy Majorana neutrinos [19,57]. This is because the $C P$ violating source terms contributing to the generation of lepton number asymmetry (i.e., the difference between the number density of leptons and that of antileptons) in the Boltzmann equations depend only on the following combinations of $C P$ asymmetries from different generations of heavy Majorana neutrinos in the limit of complete mass degeneracy [57], namely,

$\epsilon_{\mathrm{eff}} \equiv \frac{\sum_{i=1}^{3}\left[\Gamma\left(N_{i} \rightarrow \ell_{\alpha}+H\right)-\Gamma\left(N_{i} \rightarrow \bar{\ell}_{\alpha}+\bar{H}\right)\right]}{\sum_{i=1}^{3} \sum_{\alpha}\left[\Gamma\left(N_{i} \rightarrow \ell_{\alpha}+H\right)+\Gamma\left(N_{i} \rightarrow \bar{\ell}_{\alpha}+\bar{H}\right)\right]}$,

which turn out to be vanishing. This can be understood by noticing that all the heavy Majorana neutrinos in the massdegeneracy limit contribute to the generation of $C P$ asymmetries and only the effective $C P$ asymmetries defined in Eq. (3.29) play a role in leptogenesis.

To conclude, although there remain four nonvanishing $C P$ phases in the limit of complete mass degeneracy, there are actually no $C P$ asymmetries in the decays of heavy Majorana neutrinos. 


\section{Minimal seesaw model}

In this section, we examine the so-called minimal seesaw model (MSM), in which only two right-handed neutrino singlets are introduced [62-66]. See, e.g., Refs. [67,68] for recent reviews on the MSM. In this minimal scenario, $M_{\mathrm{D}}$ is actually a $3 \times 2$ complex matrix, and the effective mass matrix of three light Majorana neutrinos is given by the seesaw formula $M_{\nu}=-M_{\mathrm{D}} M_{\mathrm{R}}^{-1} M_{\mathrm{D}}^{\mathrm{T}}$. As is well known, the rank of $M_{\nu}$ will thus be at most 2, indicating that the lightest neutrino is massless. Without loss of generality, we take $m_{1}=0$ for the normal mass ordering for illustration.

Although $M_{\mathrm{D}}$ generally contains six phases, three of them are actually unphysical and can be removed by the basis transformations of lepton fields $\nu_{\mathrm{L}}, l_{\mathrm{L}}$, and $l_{\mathrm{R}}$. In the following discussions, we take the Casas-Ibarra parametrization of $M_{\mathrm{D}}[69,70]$, i.e.,

$$
M_{\mathrm{D}}=\mathrm{i} U \sqrt{\hat{M}_{\nu}} R \sqrt{\hat{M}_{\mathrm{R}}},
$$

where the PMNS matrix $U$ can be decomposed as $U=V \cdot \operatorname{diag}\left\{1, e^{\mathrm{i} \sigma}, 1\right\},{ }^{6}$ with $V$ being the CKM-like matrix that contains one Dirac $C P$ phase $\delta$ and three mixing angles. In addition, both light and heavy Majorana neutrino mass matrices $\hat{M}_{\nu}=\operatorname{diag}\left\{0, m_{2}, m_{3}\right\}$ and $\hat{M}_{\mathrm{R}}=$ $\operatorname{diag}\left\{M_{1}, M_{2}\right\}$ are diagonal, and the complex and orthogonal matrix $R$, satisfying $R^{\mathrm{T}} R=\operatorname{diag}\{1,1\}$ and $R R^{\mathrm{T}}=$ $\operatorname{diag}\{0,1,1\}$ can be parametrized as [70]

$$
R=\left(\begin{array}{cc}
0 & 0 \\
\cos z & -\sin z \\
\pm \sin z & \pm \cos z
\end{array}\right),
$$

where $z$ is an arbitrary complex number. With such a parametrization, one can observe that one $C P$ phase of $M_{\mathrm{D}}$ is located in $R$, while the other two are included in the PMNS matrix $U$.

Now we explain how to construct the WB invariants in the MSM and present the sufficient and necessary conditions for $C P$ conservation in the cases of nondegenerate (i.e., $M_{1} \neq M_{2}$ ) and degenerate (i.e., $M_{1}=M_{2}$ ) heavy Majorana neutrino masses.

(a) For $M_{1} \neq M_{2}$, there are in total three $C P$ phases in $M_{\mathrm{D}}$, for which one has to construct three WB invariants to guarantee $C P$ conservation. In the MSM, however, only two out of the six invariants in Eqs. (3.9)-(3.14) are linearly independent, and we choose $\tilde{\mathcal{I}}_{1}$ and $\tilde{\mathcal{I}}_{4}$. As one can see from the definition of $\tilde{\mathcal{I}}_{1}$ in Eq. (3.9), only $H_{\mathrm{D}}$ is involved in this invariant, so it contains the unique $C P$ phase in $R$. On the other

\footnotetext{
${ }^{6}$ As the lightest neutrino is massless, the Majorana $C P$ phase associated with the corresponding neutrino mass eigenstate disappears from the theory.
}

hand, $\tilde{\mathcal{I}}_{4}$ defined in Eq. (3.12) depend on the $C P$ phase in $R$ as well as the two $C P$ phases in the PMNS matrix $U$. For this reason, we need to construct extra WB invariants in which the $C P$ phases in $U$ are present. Unfortunately, all the invariants $\left\{\tilde{\mathcal{I}}_{7}, \tilde{\mathcal{I}}_{8}, \tilde{\mathcal{I}}_{9}, \tilde{\mathcal{I}}_{10}\right\}$ in Eqs. (3.24)-(3.27) vanish automatically in the MSM.

Inspired by the invariants $\left\{\mathcal{I}_{1}, \mathcal{I}_{2}, \mathcal{I}_{3}, \mathcal{I}_{4}\right\}$ in the effective theory, we can simply replace $M_{\nu}$ with $-M_{\mathrm{D}} M_{\mathrm{R}}^{-1} M_{\mathrm{D}}^{\mathrm{T}}$ everywhere in these invariants and then obtain four nontrivial WB invariants in the MSM, i.e.,

$$
\begin{aligned}
& \hat{\mathcal{I}}_{1} \equiv \operatorname{Tr}\left\{\left[M_{\mathrm{D}} M_{\mathrm{R}}^{-1} H_{\mathrm{D}}^{*}\left(M_{\mathrm{R}}^{-1}\right)^{\dagger} M_{\mathrm{D}}^{\dagger}, H_{l}\right]^{3}\right\} \\
& \hat{\mathcal{I}}_{2} \equiv \operatorname{Im}\left\{\operatorname { T r } \left[H_{l} M_{\mathrm{D}} M_{\mathrm{R}}^{-1} H_{\mathrm{D}}^{*}\left(M_{\mathrm{R}}^{-1}\right)^{\dagger}\right.\right. \\
&\left.\left.\times H_{\mathrm{D}} M_{\mathrm{R}}^{-1} M_{\mathrm{D}}^{T} H_{l}^{*} M_{\mathrm{D}}^{*}\left(M_{\mathrm{R}}^{-1}\right)^{\dagger} M_{\mathrm{D}}^{\dagger}\right]\right\} \\
& \hat{\mathcal{I}}_{3} \equiv \operatorname{Tr}\left\{\left[M_{\mathrm{D}} M_{\mathrm{R}}^{-1} M_{\mathrm{D}}^{\mathrm{T}} H_{l}^{*} M_{\mathrm{D}}^{*}\left(M_{\mathrm{R}}^{-1}\right)^{\dagger} M_{\mathrm{D}}^{\dagger}, H_{l}\right]^{3}\right\} \\
& \hat{\mathcal{I}}_{4} \equiv \operatorname{Im}\left\{\operatorname { T r } \left[H_{l}\left(M_{\mathrm{D}} M_{\mathrm{R}}^{-1} H_{\mathrm{D}}^{*}\left(M_{\mathrm{R}}^{-1}\right)^{\dagger} M_{\mathrm{D}}^{\dagger}\right)^{2}\right.\right. \\
&\left.\left.\times M_{\mathrm{D}} M_{\mathrm{R}}^{-1} M_{\mathrm{D}}^{\mathrm{T}} H_{l}^{*} M_{\mathrm{D}}^{*}\left(M_{\mathrm{R}}^{-1}\right)^{\dagger} M_{\mathrm{D}}^{\dagger}\right]\right\}
\end{aligned}
$$

It should be noted that $\hat{\mathcal{I}}_{i}$ (for $i=1,2,3,4$ ) depend on only two $C P$ phases in the PMNS matrix $U$ and have nothing to do with the $C P$ phase in $R$. Moreover, $\hat{\mathcal{I}}_{1}$ is proportional to $\sin \delta$, where $\delta$ is the Dirac-type $C P$ phase in the PMNS matrix $U$, but not related to the Majorana-type $C P$ phase $\sigma$. In contrast, $\left\{\hat{\mathcal{I}}_{2}, \hat{\mathcal{I}}_{3}, \hat{\mathcal{I}}_{4}\right\}$ depend on both $\delta$ and $\sigma$. With all these invariants, to guarantee $C P$ conservation, we can first require $\tilde{\mathcal{I}}_{1}=0$ to render the phase in $R$ trivial, then $\hat{\mathcal{I}}_{1}=0$ to eliminate $\delta$ in $U$, and finally either $\tilde{\mathcal{I}}_{4}=0$ or one of $\left\{\hat{\mathcal{I}}_{2}=0, \hat{\mathcal{I}}_{3}=0, \hat{\mathcal{I}}_{4}=0\right\}$ to get rid of $\sigma$ in $U$.

(b) For $M_{1}=M_{2}$, as in the case of partial mass degeneracy in the effective theory or in the canonical seesaw model, there is an extra degree of freedom in the system, which can be implemented to remove the only $C P$ phase in $R$. Therefore, we are left with two $C P$ phases. It is straightforward to verify that $\tilde{\mathcal{I}}_{1}$ and $\tilde{\mathcal{I}}_{4}$ vanish automatically in this limit of $M_{1}=M_{2}$. However, since $\left\{\hat{\mathcal{I}}_{1}, \hat{\mathcal{I}}_{2}, \hat{\mathcal{I}}_{3}, \hat{\mathcal{I}}_{4}\right\}$ are independent of heavy Majorana neutrino masses, they are in general nonzero in the presence of mass degeneracy. We can first use $\hat{\mathcal{I}}_{1}=0$ to make $\delta$ in $U$ trivial, then choose any one of $\left\{\hat{\mathcal{I}}_{2}=0, \hat{\mathcal{I}}_{3}=0, \hat{\mathcal{I}}_{4}=0\right\}$ to eliminate the remaining phase $\sigma$ in $U$ so that $C P$ conservation is guaranteed. In summary, if there is no mass degeneracy of heavy Majorana neutrinos, we have three $C P$ phases and the vanishing of three WB invariants $\left\{\tilde{\mathcal{I}}_{1}, \tilde{\mathcal{I}}_{4}, \hat{\mathcal{I}}_{1}\right\}$ serves as the sufficient and necessary condition for $C P$ conservation. In addition, in the case of mass degeneracy, there are two $C P$ phases and one can find that $C P$ conservation is ensured by $\left\{\hat{\mathcal{I}}_{1}=0, \hat{\mathcal{I}}_{2}=0\right\}$. It is worth mentioning that the choice of 
WB invariants is by no means unique, but different choices are all equivalent.

\section{SUMMARY}

In this paper, we have performed a systematic study of the sufficient and necessary conditions for $C P$ conservation in leptonic sector, both in the low-energy effective theory of massive Majorana neutrinos and in the canonical seesaw model. A particular attention has been paid to the cases of the mass degeneracy of either light or heavy Majorana neutrinos. We have demonstrated how to count correctly the number of independent $C P$ phases in these cases and have explained the strategy to construct the WB invariants to guarantee $C P$ conservation.

In the low-energy effective theory, if the masses of light Majorana neutrinos are not degenerate, there are in total three independent $C P$ phases. If the masses of light neutrinos are partially or completely degenerate, then there will be extra degrees of freedom in the theory, allowing us to rotate the left-handed neutrino fields without changing their mass term. As a consequence, such degrees of freedom can be used to reduce the number of independent $C P$ phases. The number of $C P$ phases and the WB invariants chosen to guarantee $C P$ conservation in different cases are summarized in Table I. Moreover, the renormalization-group equations of the WB invariants in the effective theory have been derived. By using these equations of WB invariants, we show that $C P$ conservation will not be violated by radiative corrections.

In the canonical seesaw model, there are in total six independent $C P$ phases in the case of nondegenerate masses of heavy Majorana neutrinos. Just as in the effective theory, in the presence of mass degeneracy, it is possible to reduce the number of $C P$ phases. The main results have been summarized in Table II. The sufficient and necessary conditions for $C P$ conservation in the minimal seesaw model are also given. In the basis where the charged-lepton

TABLE I. Summary of the number of independent $C P$ phases and the weak-basis invariants chosen to guarantee $C P$ conservation in the low-energy effective theory. Notice that the choice of weak-basis invariants is by no means unique.

\begin{tabular}{lcl}
\hline \hline $\begin{array}{l}\text { Low-energy effective } \\
\text { theory }\end{array}$ & $\begin{array}{c}\text { Number of } \\
C P \text { phases }\end{array}$ & Weak-basis invariants \\
\hline $\begin{array}{c}\text { No degeneracy } \\
\left(m_{1} \neq m_{2} \neq m_{3}\right)\end{array}$ & 3 & $\begin{array}{l}\mathcal{I}_{1} \equiv \operatorname{Tr}\left\{\left[H_{\nu}, H_{l}\right]^{3}\right\} \\
\mathcal{I}_{2} \equiv \operatorname{Im}\left\{\operatorname{Tr}\left[H_{l} H_{\nu} G_{l \nu}\right]\right\}\end{array}$ \\
$\begin{array}{ccc}\text { Partial degeneracy } \\
\left(m_{1}=m_{2} \neq m_{3}\right)\end{array}$ & 2 & $\begin{array}{l}\mathcal{I}_{4} \equiv \operatorname{Im}\left\{\operatorname{Tr}\left[H_{l} H_{\nu}^{2} G_{l \nu}\right]\right\} \\
\mathcal{I}_{2} \equiv \operatorname{Im}\left\{\operatorname{Tr}\left[H_{l} H_{\nu} G_{l l}\right]\right\}\end{array}$ \\
$\begin{array}{c}\text { Full degeneracy } \\
\left(m_{1}=m_{2}=m_{3}\right)\end{array}$ & 1 & $\begin{array}{l}\mathcal{I}_{3} \equiv \operatorname{Tr}\left\{\left[G_{l \nu}, H_{l}\right]^{3}\right\} \\
\mathcal{I}_{3} \equiv \operatorname{Tr}\left\{\left[G_{l \nu}, H_{l}\right]^{3}\right\}\end{array}$ \\
$\begin{aligned} \text { No degeneracy } \\
\text { with } m_{1}=0\end{aligned}$ & 2 & $\begin{array}{l}\mathcal{I}_{1} \equiv \operatorname{Tr}\left\{\left[H_{\nu}, H_{l}\right]^{3}\right\} \\
\mathcal{I}_{2} \equiv \operatorname{Im}\left\{\operatorname{Tr}\left[H_{l} H_{\nu} G_{l \nu}\right]\right\}\end{array}$ \\
\hline \hline
\end{tabular}

TABLE II. Summary of the number of independent $C P$ phases and the weak-basis invariants chosen to guarantee $C P$ conservation in the canonical seesaw model. Notice that the choice of weak-basis invariants is by no means unique.

\begin{tabular}{|c|c|c|}
\hline $\begin{array}{l}\text { Canonical seesaw } \\
\text { model }\end{array}$ & $\begin{array}{l}\text { Number of } \\
C P \text { phases }\end{array}$ & Weak-basis invariants \\
\hline $\begin{array}{l}\text { No degeneracy } \\
\qquad\left(M_{1} \neq M_{2} \neq M_{3}\right)\end{array}$ & 6 & $\begin{array}{l}\tilde{\mathcal{I}}_{1} \equiv \operatorname{Im}\left\{\operatorname{Tr}\left[H_{\mathrm{D}} H_{\mathrm{R}} G_{\mathrm{DR}}\right]\right\} \\
\tilde{\mathcal{I}}_{2} \equiv \operatorname{Im}\left\{\operatorname{Tr}\left[H_{\mathrm{D}} H_{\mathrm{R}}^{2} G_{\mathrm{DR}}\right]\right\} \\
\tilde{\mathcal{I}}_{3}^{\prime} \equiv \operatorname{Tr}\left\{\left[H_{\mathrm{R}}, H_{\mathrm{D}}\right]^{3}\right\} \\
\tilde{\mathcal{I}}_{4} \equiv \operatorname{Im}\left\{\operatorname{Tr}\left[H_{1} H_{\mathrm{R}} G_{1}\right]\right\} \\
\tilde{\mathcal{I}}_{5} \equiv \operatorname{Im}\left\{\operatorname{Tr}\left[H_{1} H_{\mathrm{R}}^{2} G_{1}\right]\right\} \\
\tilde{\mathcal{I}}_{6} \equiv \operatorname{Im}\left\{\operatorname{Tr}\left[H_{1} H_{\mathrm{R}} G_{1} H_{\mathrm{R}}\right]\right\}\end{array}$ \\
\hline $\begin{array}{l}\text { Partial degeneracy } \\
\qquad\left(M_{1}=M_{2} \neq M_{3}\right)\end{array}$ & 5 & $\begin{array}{l}\tilde{\mathcal{I}}_{1} \equiv \operatorname{Im}\left\{\operatorname{Tr}\left[H_{\mathrm{D}} H_{\mathrm{R}} G_{\mathrm{DR}}\right]\right\} \\
\tilde{\mathcal{I}}_{7} \equiv \operatorname{Tr}\left\{\left[G_{\mathrm{DR}}, H_{\mathrm{D}}\right]^{3}\right\} \\
\tilde{\mathcal{I}}_{8} \equiv \operatorname{Tr}\left\{\left[G_{1}, H_{1}\right]^{3}\right\} \\
\tilde{\mathcal{I}}_{9} \equiv \operatorname{Tr}\left\{\left[G_{2}, H_{2}\right]^{3}\right\} \\
\tilde{\mathcal{I}}_{10} \equiv \operatorname{Tr}\left\{\left[G_{3}, H_{3}\right]^{3}\right\}\end{array}$ \\
\hline $\begin{array}{l}\text { Full degeneracy } \\
\qquad\left(M_{1}=M_{2}=M_{3}\right)\end{array}$ & 4 & $\begin{array}{l}\tilde{\mathcal{I}}_{7} \equiv \operatorname{Tr}\left\{\left[G_{\mathrm{DR}}, H_{\mathrm{D}}\right]^{3}\right\} \\
\tilde{\mathcal{I}}_{8} \equiv \operatorname{Tr}\left\{\left[G_{1}, H_{1}\right]^{3}\right\} \\
\tilde{\mathcal{I}}_{9} \equiv \operatorname{Tr}\left\{\left[G_{2}, H_{2}\right]^{3}\right\} \\
\tilde{\mathcal{I}}_{10} \equiv \operatorname{Tr}\left\{\left[G_{3}, H_{3}\right]^{3}\right\}\end{array}$ \\
\hline $\begin{array}{l}\text { Minimal seesaw } \\
\quad \text { model }\left(M_{1} \neq M_{2}\right)\end{array}$ & 3 & $\begin{array}{l}\tilde{\mathcal{I}}_{1} \equiv \operatorname{Im}\left\{\operatorname{Tr}\left[H_{\mathrm{D}} H_{\mathrm{R}} G_{\mathrm{DR}}\right]\right\} \\
\tilde{\mathcal{I}}_{4} \equiv \operatorname{Im}\left\{\operatorname{Tr}\left[H_{1} H_{\mathrm{R}} G_{1}\right]\right\} \\
\hat{\mathcal{I}}_{1} \text { in Eq. }(3.32)\end{array}$ \\
\hline $\begin{array}{l}\text { Minimal seesaw } \\
\quad \text { model }\left(M_{1}=M_{2}\right)\end{array}$ & 2 & $\begin{array}{l}\hat{\mathcal{I}}_{1} \text { in Eq. }(3.32) \\
\hat{\mathcal{I}}_{2} \text { in Eq. }(3.33)\end{array}$ \\
\hline
\end{tabular}

mass matrix $M_{l}$ and right-handed neutrino mass matrix $M_{\mathrm{R}}$ are diagonal, the conserved $C P$ symmetry would lead to the vanishing of all flavor-dependent $C P$ asymmetries in the heavy Majorana neutrino decays, i.e., $\epsilon_{i \alpha}$ for $i=1,2,3$ and $\alpha=e, \mu, \tau$, while any nonzero $C P$ asymmetries imply the existence of $C P$ violation. It is worth pointing out that a flavor symmetry must be introduced to protect the mass degeneracy. Otherwise, either partial or complete mass degeneracy of heavy Majorana neutrinos will be violated by radiative corrections [51-56] and all six WB invariants in Eqs. (3.9)-(3.14) are needed to guarantee $C P$ conservation.

We stress that the choice of different sets of WB invariants for $C P$ conservation is not unique. In each case, we have explicitly given a suitable set of WB invariants, which should be useful for the future studies of leptonic $C P$ violation and for the model building of neutrino mass generation and lepton flavor mixing.

\section{ACKNOWLEDGMENTS}

The authors thank Professors Thomas Schwetz and Zhizhong Xing for helpful discussions. This work was supported in part by the National Natural Science Foundation of China under Grants No. 11775232 and No. 11835013, and by the CAS Center for Excellence in Particle Physics. 
[1] M. Tanabashi et al. (Particle Data Group), Review of particle physics, Phys. Rev. D 98, 030001 (2018).

[2] Z.z. Xing, Flavor structures of charged fermions and massive neutrinos, Phys. Rep. 854, 1 (2020).

[3] G. C. Branco, R. G. Felipe, and F. R. Joaquim, Leptonic CP violation, Rev. Mod. Phys. 84, 515 (2012).

[4] E. Majorana, A symmetric theory of electrons and positrons, Nuovo Cimento 14, 171 (1937).

[5] G. Racah, On the symmetry of particle and antiparticle, Nuovo Cimento 14, 322 (1937).

[6] P. Minkowski, $\mu \rightarrow e \gamma$ at a rate of one out of $10^{9}$ muon decays?, Phys. Lett. 67B, 421 (1977).

[7] T. Yanagida, Horizontal symmetry and masses of neutrinos, Conf. Proc. C7902131, 95 (1979).

[8] M. Gell-Mann, P. Ramond, and R. Slansky, Complex spinors and unified theories, Conf. Proc. C790927, 315 (1979).

[9] S. L. Glashow, The future of elementary particle physics, NATO Adv. Study Inst. Ser., Ser. B 61, 687 (1980).

[10] R. N. Mohapatra and G. Senjanovic, Neutrino Mass and Spontaneous Parity Violation, Phys. Rev. Lett. 44, 912 (1980).

[11] B. Pontecorvo, Mesonium and anti-mesonium, Zh. Eksp. Teor. Fiz. 33, 549 (1957) [Sov. Phys. JETP 6, 429 (1957)], http://www.jetp.ac.ru/cgi-bin/e/index/e/6/2/p429?a=list.

[12] Z. Maki, M. Nakagawa, and S. Sakata, Remarks on the unified model of elementary particles, Prog. Theor. Phys. 28, 870 (1962).

[13] A. D. Sakharov, Violation of $C P$ invariance, C asymmetry, and baryon asymmetry of the Universe, Pis'ma Zh. Eksp. Teor. Fiz. 5, 32 (1967) [JETP Lett. 5, 24 (1967)].

[14] M. Fukugita and T. Yanagida, Baryogenesis without grand unification, Phys. Lett. B 174, 45 (1986).

[15] S. Davidson, E. Nardi, and Y. Nir, Leptogenesis, Phys. Rep. 466, 105 (2008).

[16] D. Bodeker and W. Buchmuller, Baryogenesis from the weak scale to the GUT scale, arXiv:2009.07294.

[17] G. C. Branco, L. Lavoura, and M. N. Rebelo, Majorana neutrinos and $C P$ violation in the leptonic sector, Phys. Lett. B 180, 264 (1986).

[18] J. Bernabeu, G. C. Branco, and M. Gronau, $C P$ restrictions on quark mass matrices, Phys. Lett. 169B, 243 (1986).

[19] A. Pilaftsis, $C P$ violation and baryogenesis due to heavy Majorana neutrinos, Phys. Rev. D 56, 5431 (1997).

[20] G. C. Branco, T. Morozumi, B. M. Nobre, and M. N. Rebelo, A bridge between $C P$ violation at low-energies and leptogenesis, Nucl. Phys. B617, 475 (2001).

[21] V. Cirigliano, G. Isidori, and V. Porretti, $C P$ violation and leptogenesis in models with minimal lepton flavour violation, Nucl. Phys. B763, 228 (2007).

[22] H. K. Dreiner, J. S. Kim, O. Lebedev, and M. Thormeier, Supersymmetric Jarlskog invariants: The neutrino sector, Phys. Rev. D 76, 015006 (2007).

[23] B. Yu and S. Zhou, The number of sufficient and necessary conditions for $C P$ conservation with Majorana neutrinos: Three or four?, Phys. Lett. B 800, 135085 (2020).

[24] G. C. Branco, M. N. Rebelo, and J. I. Silva-Marcos, Degenerate and Quasidegenerate Majorana Neutrinos, Phys. Rev. Lett. 82, 683 (1999).
[25] J. w. Mei and Z. z. Xing, Impact of fermion mass degeneracy on flavor mixing, J. Phys. G 30, 1243 (2004).

[26] M. N. Rebelo, Neutrino physics and leptonic weak basis invariants, arXiv:1804.05777.

[27] C. Jarlskog, Commutator of the Quark Mass Matrices in the Standard Electroweak Model and a Measure of Maximal CP Violation, Phys. Rev. Lett. 55, 1039 (1985).

[28] D. d. Wu, The rephasing invariants and $C P$, Phys. Rev. D 33, 860 (1986).

[29] H. Y. Cheng, Kobayashi-Maskawa type of hard $C P$ violation model with three generation Majorana neutrinos, Phys. Rev. D 34, 2794 (1986).

[30] T. Feldmann, T. Mannel, and S. Schwertfeger, Renormalization group evolution of flavour invariants, J. High Energy Phys. 10 (2015) 007.

[31] P. H. Chankowski and Z. Pluciennik, Renormalization group equations for seesaw neutrino masses, Phys. Lett. B 316, 312 (1993).

[32] K. S. Babu, C. N. Leung, and J. T. Pantaleone, Renormalization of the neutrino mass operator, Phys. Lett. B 319, 191 (1993).

[33] S. Antusch, M. Drees, J. Kersten, M. Lindner, and M. Ratz, Neutrino mass operator renormalization revisited, Phys. Lett. B 519, 238 (2001).

[34] S. Antusch, J. Kersten, M. Lindner, and M. Ratz, Neutrino mass matrix running for nondegenerate seesaw scales, Phys. Lett. B 538, 87 (2002).

[35] S. Antusch, J. Kersten, M. Lindner, and M. Ratz, Running neutrino masses, mixings and $C P$ phases: Analytical results and phenomenological consequences, Nucl. Phys. B674, 401 (2003).

[36] J. w. Mei and Z. z. Xing, Radiative corrections to neutrino mixing and $C P$ violation in the minimal seesaw model with leptogenesis, Phys. Rev. D 69, 073003 (2004).

[37] S. Antusch, J. Kersten, M. Lindner, M. Ratz, and M. A. Schmidt, Running neutrino mass parameters in see-saw scenarios, J. High Energy Phys. 03 (2005) 024.

[38] J. w. Mei, Running neutrino masses, leptonic mixing angles and $C P$-violating phases: From $M_{\mathrm{Z}}$ to $\Lambda_{\mathrm{GUT}}$, Phys. Rev. D 71, 073012 (2005).

[39] S. Luo, J. w. Mei, and Z.z. Xing, Radiative generation of leptonic $C P$ violation, Phys. Rev. D 72, 053014 (2005).

[40] Z. z. Xing, A novel parametrization of tau-lepton dominance and simplified one-loop renormalization-group equations of neutrino mixing angles and $C P$-violating phases, Phys. Lett. B 633, 550 (2006).

[41] Z.z. Xing and S. Zhou, Neutrinos in Particle Physics, Astronomy and Cosmology (Springer-Verlag, Berlin, 2011).

[42] T. Ohlsson, H. Zhang, and S. Zhou, Radiative corrections to the leptonic Dirac CP-violating phase, Phys. Rev. D 87, 013012 (2013).

[43] T. Ohlsson and S. Zhou, Renormalization group running of neutrino parameters, Nat. Commun. 5, 5153 (2014).

[44] Z.z. Xing, D. Zhang, and J. y. Zhu, The $\mu$ - $\tau$ reflection symmetry of Dirac neutrinos and its breaking effect via quantum corrections, J. High Energy Phys. 11 (2017) 135.

[45] Z. z. Xing and S. Zhou, Naumov- and Toshev-like relations in the renormalization-group evolution of quarks and Dirac neutrinos, Chin. Phys. C 42, 103105 (2018). 
[46] J. Y. Zhu, Leptonic unitarity triangles: RGE running effects and $\mu-\tau$ reflection symmetry breaking, Phys. Rev. D 99, 033003 (2019).

[47] D. Zhang, Integral solutions to the one-loop renormalization-group equations for lepton flavor mixing parameters and the Jarlskog invariant, arXiv:2007.12976.

[48] I. Esteban, M. C. Gonzalez-Garcia, M. Maltoni, T. Schwetz, and A. Zhou, The fate of hints: Updated global analysis of three-flavor neutrino oscillations, J. High Energy Phys. 09 (2020) 178.

[49] F. Capozzi, E. Lisi, A. Marrone, and A. Palazzo, Current unknowns in the three neutrino framework, Prog. Part. Nucl. Phys. 102, 48 (2018).

[50] T. Endoh, T. Morozumi, T. Onogi, and A. Purwanto, $C P$ violation in seesaw model, Phys. Rev. D 64, 013006 (2001); Erratum, Phys. Rev. D 64, 059904 (2001).

[51] R. G. Felipe, F. R. Joaquim, and B. M. Nobre, Radiatively induced leptogenesis in a minimal seesaw model, Phys. Rev. D 70, 085009 (2004).

[52] K. Turzynski, Degenerate minimal seesaw and leptogenesis, Phys. Lett. B 589, 135 (2004).

[53] F. R. Joaquim, Radiative leptogenesis in minimal seesaw models, Nucl. Phys. B, Proc. Suppl. 145, 276 (2005).

[54] G. C. Branco, R. G. Felipe, F. R. Joaquim, and B. M. Nobre, Enlarging the window for radiative leptogenesis, Phys. Lett. B 633, 336 (2006).

[55] P. S. B. Dev, P. Millington, A. Pilaftsis, and D. Teresi, Flavour covariant transport equations: An application to resonant leptogenesis, Nucl. Phys. B897, 749 (2015).

[56] A. Pilaftsis and D. Teresi, Mass bounds on light and heavy neutrinos from radiative minimal-flavor-violation leptogenesis, Phys. Rev. D 92, 085016 (2015).

[57] A. Pilaftsis and T. E. J. Underwood, Resonant leptogenesis, Nucl. Phys. B692, 303 (2004).

[58] A. Pilaftsis and T.E. J. Underwood, Electroweak-scale resonant leptogenesis, Phys. Rev. D 72, 113001 (2005).
[59] A. Anisimov, A. Broncano, and M. Plumacher, The $C P$ asymmetry in resonant leptogenesis, Nucl. Phys. B737, 176 (2006).

[60] B. Dev, M. Garny, J. Klaric, P. Millington, and D. Teresi, Resonant enhancement in leptogenesis, Int. J. Mod. Phys. A 33, 1842003 (2018).

[61] J. Zhang and S. Zhou, A further study of the FramptonGlashow-Yanagida model for neutrino masses, flavor mixing and baryon number asymmetry, J. High Energy Phys. 09 (2015) 065.

[62] A. Kleppe, Extending the standard model with two right-handed neutrinos, in Proceedings of the 3rd Tallinn Symposium on Neutrino Physics, Lohusalu, Estonia, (1995) pp. 118-125, https://www.osti.gov/etdeweb/biblio/493672.

[63] E. Ma, D. P. Roy, and U. Sarkar, A seesaw model for atmospheric and solar neutrino oscillations, Phys. Lett. B 444, 391 (1998).

[64] S. F. King, Large mixing angle MSW and atmospheric neutrinos from single right-handed neutrino dominance and U(1) family symmetry, Nucl. Phys. B576, 85 (2000).

[65] S. F. King, Constructing the large mixing angle MNS matrix in seesaw models with right-handed neutrino dominance, J. High Energy Phys. 09 (2002) 011.

[66] P. H. Frampton, S. L. Glashow, and T. Yanagida, Cosmological sign of neutrino $C P$ violation, Phys. Lett. B 548, 119 (2002).

[67] W. 1. Guo, Z. z. Xing, and S. Zhou, Neutrino masses, lepton flavor mixing and leptogenesis in the minimal seesaw model, Int. J. Mod. Phys. E 16, 1 (2007).

[68] Z. z. Xing and Z. h. Zhao, The minimal seesaw and leptogenesis models, arXiv:2008.12090.

[69] J. A. Casas and A. Ibarra, Oscillating neutrinos and $\mu \rightarrow e \gamma$, Nucl. Phys. B618, 171 (2001).

[70] A. Ibarra and G. G. Ross, Neutrino phenomenology: The case of two right-handed neutrinos, Phys. Lett. B 591, 285 (2004). 